\title{
UK Iatrogenic Creutzfeldt-Jakob disease: investigating human prion transmission across genotypic barriers using human tissue-based and molecular approaches
}

\author{
Diane L. Ritchie ${ }^{1} \cdot$ Marcelo A. Barria $^{1} \cdot$ Alexander H. Peden $^{1} \cdot$ Helen M. Yull ${ }^{1}$ \\ James Kirkpatrick $^{1} \cdot$ Peter Adlard $^{2} \cdot$ James W. Ironside $^{1} \cdot$ Mark W. Head $^{1,3}(\mathbb{C}$
}

Received: 10 September 2016 / Revised: 20 October 2016 / Accepted: 25 October 2016 / Published online: 3 November 2016

(c) The Author(s) 2016. This article is published with open access at Springerlink.com

\begin{abstract}
Creutzfeldt-Jakob disease (CJD) is the prototypic human prion disease that occurs most commonly in sporadic and genetic forms, but it is also transmissible and can be acquired through medical procedures, resulting in iatrogenic CJD (iCJD). The largest numbers of iCJD cases that have occurred worldwide have resulted from contaminated cadaveric pituitary-derived human growth hormone $(\mathrm{hGH})$ and its use to treat primary and secondary growth hormone deficiency. We report a comprehensive, tissuebased and molecular genetic analysis of the largest series of UK hGH-iCJD cases reported to date, including in vitro kinetic molecular modelling of genotypic factors influencing prion transmission. The results show the interplay of prion strain and host genotype in governing the molecular, pathological and temporal characteristics of the UK hGH-iCJD epidemic and provide insights into the adaptive mechanisms involved when prions cross genotypic barriers. We conclude that all of the available evidence is consistent with the hypothesis that the UK hGH-iCJD epidemic
\end{abstract}

D. L. Ritchie and M. A. Barria contributed equally to this work.

Electronic supplementary material The online version of this article (doi:10.1007/s00401-016-1638-x) contains supplementary material, which is available to authorized users.

Mark W. Head

m.w.head@ed.ac.uk

1 National CJD Research \& Surveillance Unit, Centre for Clinical Brain Sciences, Deanery of Clinical Sciences, The University of Edinburgh, Edinburgh, UK

2 University College London Institute of Child Health, 30 Guilford Street, London WC1N 1EH, UK

3 National CJD Research \& Surveillance Unit, University of Edinburgh, Bryan Matthews Building, Western General Hospital, Edinburgh EH4 2XU, UK resulted from transmission of the V2 human prion strain, which is associated with the second most common form of sporadic CJD.

Keywords Creutzfeldt-Jakob disease · Prion · Iatrogenic · Growth hormone $\cdot$ Disease phenotype $\cdot$ Agent strain

\section{Introduction}

Prion diseases are a group of fatal transmissible neurodegenerative disorders that occur naturally in man and a range of other species [12]. All are associated with the conversion of a normal host cellular protein, the prion protein $\left(\operatorname{PrP}^{\mathrm{C}}\right)$, into a misfolded disease-associated form, $\mathrm{PrP}^{\mathrm{Sc}}$, which accumulates in the central nervous system (CNS) [29]. The prion hypothesis states that $\operatorname{PrP}^{\mathrm{Sc}}$ is the principal (if not the sole) component of the transmissible agents responsible for this group of diseases [30]; this hypothesis is supported by an increasing body of experimental evidence [9]. However, the precise relationship between the conformation of $\operatorname{PrP}^{\mathrm{Sc}}$ in different forms of prion disease and the biological properties of the transmissible agents (strain characteristics) in different prion diseases is still unresolved.

Unlike the more common forms of human neurodegenerative diseases, human prion diseases occur in sporadic, genetic and acquired forms [12]. The acquired human prion diseases that have resulted from human-to-human transmission include kuru (now considered to be extinct) and iatrogenic Creutzfeldt-Jakob disease (iCJD), with variant Creutzfeldt-Jakob disease (vCJD) resulting from bovine to human transmission of the bovine spongiform encephalopathy (BSE) agent. Iatrogenic transmission of Creutzfeldt-Jakob disease (CJD) has occurred in many countries across the world as a consequence of transmission 
via contaminated neurosurgical instruments, intracerebral electroencephalography needles, corneal transplantation, human dura mater grafts, inoculation with human pituitary hormones and (in the case of vCJD) transfusion of packed red blood cells [14]. The vast majority of iCJD cases have occurred in human dura mater (hDM) graft recipients and in recipients of human growth hormone (hGH) derived from cadaveric pituitaries [5]. The incubation periods in the worldwide cohort of iCJD cases are markedly variable, with the shortest incubation periods occurring in those exposed via surgical instruments or electrodes used on the CNS (1-2.3 years) and the longest occurring in $\mathrm{hDM}$ graft recipients (1.3-30 years), and human pituitary-derived hGH and gonadotrophin recipients (5-42 years) [5]. Estimation of incubation periods in human pituitary hormone recipients is difficult, since the patients are often treated over a period of years; the time period from the mid-point of pituitary hormone treatment to the onset of clinical symptoms of iCJD is often used as an estimate for the incubation period [33].

The use of pituitary-derived hGH in the treatment of primary and secondary growth hormone deficiency in the United Kingdom (UK) began as a clinical trial in 1959 and became a centrally administered National Health Service activity in 1976 [33]. In 1985, the first cases of iCJD in patients treated with pituitary-derived hGH were reported in the United States of America (USA) and in the UK [10, 28]. Consequently, the use of pituitary-derived hGH for the treatment of growth hormone deficiency was abandoned in the UK, USA and many other countries and biosynthetic growth hormone is now used for this purpose. The presence of prion infectivity in hGH preparations in the USA was confirmed by experimental intracerebral inoculation of samples of pituitary-derived hGH into primates, which resulted in the onset of neurological symptoms in a squirrel monkey after an incubation period of over 5.5 years [11]. Post-mortem examination of the brain of this animal confirmed the presence of a subacute spongiform encephalopathy.

In the UK, 1849 patients were treated with hGH in the national scheme from 1959 until 1985 [33]. Since 1985, 78 deaths from iatrogenic CJD have occurred in this cohort, and it has been identified that one particular preparation of UK pituitary-derived hGH (the Hartree-modified Wilhelmi preparation) had been administered to all hGH recipients who had developed iCJD [31, 33]. It has also been estimated that the risk of developing iCJD in the UK hGH recipient cohort was greatest in those patients who received treatment at ages 8-10 years with a peak incubation period of 20 years [33]. Estimated incubation periods in the UK hGH-iCJD patients range from 7 to 40 years [31]; these prolonged incubation periods are reminiscent of those occurring in kuru, where incubation periods of over 40 years have been reported [8]. In contrast, only eight cases of iCJD in hDM graft recipients (hDM-iCJD) have been reported in the UK, with incubation periods ranging from 3.8 to 14.8 years [13].

Incubation periods in prion diseases have been studied extensively under experimental conditions and appear to be significantly influenced by both recipient (host) genetic factors and the strain properties of the infectious agent [12]. The principal human genetic factor influencing this incubation period is the codon 129 polymorphism in the prion protein gene $(P R N P)$ of the recipient. It has long been recognised that homozygosity at this locus may predispose to both iCJD and sporadic CJD (SCJD), but differences in the frequency of the three possible genotypes (MM, MV and VV) have been reported in hGH-iCJD cohorts in different countries; in particular, most cases of hGH-iCJD in France have belonged to the MM subgroup, whilst in the UK the VV and MV subgroups predominate [4]. These differences may also reflect the nature of the strain(s) of CJD (presumably SCJD) that were responsible for the iatrogenic disease transmission.

Despite the fact that $\mathrm{iCJD}$ in $\mathrm{hGH}$ recipients has occurred in over 200 patients across the world, there have been very few detailed studies of the neuropathology, prion protein biochemistry and prion protein seeding activity in this form of human prion disease. Most reports describe only small numbers of cases that are not necessarily representative of the cohort in question, particularly with respect to the year of diagnosis in relation to the evolving time period since iatrogenic exposure to contaminated $\mathrm{hGH}$ ceased. We now report a detailed study of the largest cohort of hGH-iCJD cases reported to date, with detailed characterisation of $\operatorname{PrP}^{\mathrm{Sc}}$ in the brain, correlation with genetic and pathological factors and an exploration of the in vitro PrP seeding activity present in different genetic subgroups. These cases are compared with a limited number of cases of hDM-iCJD in the UK, but also a very much larger group of SCJD cases drawn from the UK population over a similar time period. The detailed examination of these cohorts has allowed us a unique opportunity to ask fundamental questions about person-to-person transmission of prion disease and the molecular mechanisms involved.

\section{Materials and methods}

\section{Cases, inclusion criteria and tissue specimens}

All CJD cases examined were of UK origin and were referred to the National CJD Research and Surveillance Unit (NCJDRSU) between 1991 and 2015. Diagnoses were made according to WHO criteria [35]. Inclusion criteria for biochemical investigation of iCJD cases were the availability of frozen CNS tissue taken at post-mortem, appropriate 
consent and ethical approval for retention and research use and the availability of basic clinical data. All patients referred to the NCJDRSU with a diagnosis of CJD who had received hGH therapy or a hDM graft and met these criteria were included in the study. The study identification number and basic patient demographic data for $21 \mathrm{hGH}-\mathrm{iCJD}$ and three hDM-iCJD cases are detailed in Online Resource Table 1 . Where available, samples of both frozen grey matter and the corresponding fixed samples from the cerebral cortex (frontal, parietal, temporal and occipital cortex), cerebellum, thalamus and spinal cord were examined. Data from a larger subset of the UK hGH-iCJD cases, including some of those for which frozen tissues were not available were included in genetic analyses. Data from 1080 unselected cases of UK sCJD of known PRNP codon 129, with deaths between 1990 and 2015 were used for genetic comparison with the UK hGH-iCJD cases. A smaller group of UK sCJD cases $(n=108)$ that were referred to the NCJDRSU during the period 1990-2011 were used for biochemical comparison with the hGH-iCJD group. These cases were selected on the basis of consent for research use, availability of clinical information and sufficient frozen tissue available (minimal set comprising temporal cortex, occipital cortex, parietal cortex and thalamus) for $\mathrm{PrP}^{\mathrm{res}}$ type classification according to the method of Parchi et al. [23]. All CJD tissue samples were provided by request from the NCJDRSU Brain and Tissue Bank in Edinburgh, $\mathrm{UK}$, which is part of the MRC Edinburgh Brain Bank.

\section{Neuropathological analysis}

Formalin fixed, formic acid treated, paraffin embedded tissue samples from the frontal, parietal, occipital and temporal cortices, hippocampus, amygdala, basal ganglia, thalamus, brainstem, cerebellum and spinal cord (where available) were stained with haematoxylin and eosin (H\&E) and immunohistochemistry carried out for the prion protein. Immunohistochemistry was performed using two monoclonal antibodies recognising the prion protein; 12F10/epitope: amino acids 142-160 (Bioquote Ltd, York, UK) and KG9/ epitope: amino acids 140-160 (TSE Resource Centre, Roslin Institute, UK) in combination with the highly sensitive Novolink $^{\mathrm{TM}}$ Polymer Detection System (Leica Biosystems, UK; Product RE7280-K). Briefly, $5 \mu \mathrm{m}$ paraffin embedded tissue sections were autoclaved in distilled water $\left(\mathrm{dH}_{2} \mathrm{O}\right)$ at $121{ }^{\circ} \mathrm{C}$ for $10 \mathrm{~min}$ followed by immersion in $96 \%$ formic acid for $5 \mathrm{~min}$. Tissue sections were thoroughly washed in water before incubating in proteinase $\mathrm{K}$ solution $(5 \mu \mathrm{g} /$ $\mathrm{ml}$ ) for $5 \mathrm{~min}$. Sections were washed in Tris-buffered saline (TBS) $[50 \mathrm{mM}$ Tris; $150 \mathrm{mM} \mathrm{NaCl}$; $\mathrm{pH} 7.6]$ before incubating with the primary antibodies $(12 \mathrm{~F} 10,30 \mathrm{ng} / \mathrm{ml} ; \mathrm{KG} 9$, $40 \mathrm{ng} / \mathrm{ml}$ ) diluted in antibody diluent (Leica Biosystems, UK: Product RE7133-CE) for $1 \mathrm{~h}$ at room temperature.
After $3 \times 5$ min washes in TBS, immunolabeling was completed using the Novolink ${ }^{\mathrm{TM}}$ Polymer Detection System. After a final wash, staining was visualised with 3, 3'-diaminobenzidine (DAB).

Tissue sections were analysed independently by two experienced assessors (DLR and JWI). The distribution, severity and nature of the spongiform change, neuronal loss, gliosis and amyloid plaque formation were assessed on $\mathrm{H} \& \mathrm{E}$ sections and scored in a semiquantitative manner (0-absent, 1-mild, 2-moderate, 3-severe) [23]. The distribution, severity and nature of the abnormal PrP accumulation detected by immunohistochemistry were also recorded [12]. This allowed subclassification by histotyping [23, 25] to determine whether the range of neuropathological phenotypes identified in the iCJD patients corresponded to those occurring in the recognised SCJD subtypes, or not.

\section{Western blot analysis and classification of $\operatorname{PrP}^{\text {res }}$ types}

$\mathrm{PrP}^{\mathrm{res}}$ types present in brain samples from the hGH-iCJD, hDM-iCJD and sCJD cases were determined using the refined Western blot protocol of Parchi et al. [23]. Briefly, $100 \mathrm{mg}$ samples of frozen CNS samples were subjected to stringent sample preparation (including strong buffering to pH6. 9 at $37{ }^{\circ} \mathrm{C}$ ) and digestion with proteinase $\mathrm{K}$ (Roche, Lewes, UK) using $10 \mathrm{U} / \mathrm{ml}$ corresponding to $\sim 200 \mu \mathrm{g} / \mathrm{ml}$ (depending on the batch) at $37{ }^{\circ} \mathrm{C}$ for $1 \mathrm{~h}$ prior to Western blot analysis. Immunodetection of PrP was carried out using monoclonal antibody 3F4 (Millipore, Watford, UK; Catalogue number MAB1564) at a final concentration of $75 \mathrm{ng} / \mathrm{mL}$ for $1 \mathrm{~h}$, or the monoclonal antibody 12B2 (Central Veterinary Institute of Wageningen UR, Lelystad, The Netherlands) at a final concentration of $200 \mathrm{ng} / \mathrm{ml}$ for $1 \mathrm{~h}$. This protocol allows a distinction to be made between $\mathrm{PrP}^{\mathrm{res}}$ types based on the migration of the unglycosylated (bottom) band of the typical triplet glycoform pattern. Three different unglycosylated $\mathrm{PrP}^{\mathrm{res}}$ fragment mobilities are recognised within the 21-19 kDa range: type 1 in which the unglycosylated $\mathrm{PrP}^{\mathrm{res}}$ has a molecular mass of $\sim 21 \mathrm{kDa}$, type 2 in which the unglycosylated $\mathrm{PrP}^{\mathrm{res}}$ fragment has a molecular mass of $\sim 19 \mathrm{kDa}$, and an additional type with a mobility of $\sim 20 \mathrm{kDa}$ migrating between type 1 and 2, and therefore referred to as type i (for intermediate). $\mathrm{PrP}^{\mathrm{res}}$ types $1, \mathrm{i}$ and 2 are not mutually exclusive. Certain individual samples and cases contain more than one $\mathrm{PrP}^{\mathrm{res}}$ type. This diversity is recognised by classifying such samples and cases as $1+2$ or $\mathrm{i}+2$.

\section{Real-time quaking induced conversion (RT-QuIC)}

The method used for the RT-QuIC in vitro conversion assay was the one used previously $[26,27]$ with minor modifications. Full length hamster recombinant $\operatorname{PrP}$ (recPrP, 
aa 23-231; GenBank accession no. K02234) was used as a substrate for seeded conversion. The hamster Prnp sequence encodes methionine at the position equivalent to codon 129 of the human PRNP gene. Brain samples to seed the reactions were prepared by homogenizing frozen, preweighed cerebral cortex tissue samples in phosphate buffered saline (PBS) containing $1 \mathrm{mM}$ EDTA, $150 \mathrm{mM} \mathrm{NaCl}$, $0.5 \%$ Triton X-100 and Complete ${ }^{\mathrm{TM}}$ Mini EDTA-free Protease Inhibitor Cocktail (Roche). The tissue was disrupted using one cycle of lysis (45 s, $6 \mathrm{~ms}^{-1}$ ) in a FastPrep-24 homogeniser (MP Biomedicals, Santa Ana, CA, USA). An appropriate volume of homogenization buffer was used to give a final tissue concentration of $10 \%(\mathrm{w} / \mathrm{v})$. Cellular debris was cleared from the homogenates by centrifugation at $5200 \mathrm{~g}$ at $4{ }^{\circ} \mathrm{C}$ for $5 \mathrm{~m}$. Further serial tenfold dilutions of the cleared tissue homogenate were made using PBS. The $100 \mathrm{ml}$ RT-QuIC reactions were set up in quadruplicate in the wells of a clear-bottomed black 96-well microplate (Fisher Scientific, Loughborough, UK). Stock solutions containing the reaction components were filtered through a $0.22 \mu \mathrm{M}$ Millex PES filter prior to making a master mix. recPrP was the final addition to the master mix, which was mixed by gently inverting the tube and then dispensed into the wells in $98 \mu \mathrm{l}$ volumes. At least four replicate analyses were performed on each patient sample. The RT-QuIC reactions (final volume $=100 \mu \mathrm{l}$, recPrP final concentration $=0.1 \mathrm{mg} / \mathrm{ml}$ ) were initiated by the addition of $2 \mu \mathrm{l}$ of a $10^{3}$-fold dilution of the $10 \%(\mathrm{w} / \mathrm{v})$ homogenate, which is equivalent to $2 \times 10^{-7} \mathrm{~g}$ brain (wet mass) per $100 \mu \mathrm{l}$ reaction. After adding this volume of diluted brain homogenate, the final reactions contained PBS, $324 \mathrm{mM} \mathrm{NaCl}$ (including the $\mathrm{NaCl}$ present in PBS), $1 \mathrm{mM}$ EDTA, $10 \mathrm{mM}$ ThT, $1 \times 10^{-7} \%$ Triton $^{\mathrm{TM}} \mathrm{X}-100$ and $0.1 \mathrm{mg} / \mathrm{ml} \mathrm{recPrP}$. The plates were sealed with film, incubated at $42{ }^{\circ} \mathrm{C}$ and shaken intermittently (87 s shaking at $900 \mathrm{rpm}$ in a double orbital configuration followed by a 33 s rest) using a FLUOstar OMEGA microplate reader (BMG Labtech). Fluorescence readings were taken at $480 \mathrm{~nm}$ every $15 \mathrm{~min}$ from the bottom of the wells after excitation with 20 flashes per well at $450 \mathrm{~nm}$. To quantify prion seeding efficiency we used a method based on the one described by Shi et al. [32]. In this method, the lag time to conversion is used as an inverse measure of seeding potential. The lag period to the start of conversion was taken to be the time (in hours) from the start of the assay to when the RT-QuIC fluorescence reading was three times the average reading at $0.5 \mathrm{~h}$ (the baseline). A mean lag period for four replicate analyses was thus obtained for each patient.

\section{Protein misfolding cyclic amplification}

Normal brain homogenates, from humanised transgenic mice of the PRNP codon 129 methionine homozygous
(129MM) and valine homozygous (129VV) genotypes, were prepared using a manual homogenizer and conversion buffer (Phosphate-Buffered Saline 1X, $150 \mathrm{mM}$ $\mathrm{NaCl}, 1 \%$ Triton $\mathrm{X}-100$ and $1 \mathrm{X}$ protease inhibitors) to obtain a final $10 \%$ weight/volume solution (termed the PMCA "substrate"). The homogenized tissue was cleared by centrifugation at $2000 \mathrm{rpm}$ for $40 \mathrm{~s}$ in a refrigerated centrifuge $\left(4{ }^{\circ} \mathrm{C}\right)$, and the supernatant was aliquoted and stored at $-80{ }^{\circ} \mathrm{C}$. The CJD brain homogenates (termed PMCA "seeds") were prepared following the same method used for substrate preparation. PMCA experiments were carried out as described previously [1]. Briefly, aliquots of $10 \%$ brain homogenate substrate were mixed with $10 \%$ CJD brain homogenates seeds in PCR tubes. Low molecular weight heparin was included at $100 \mu \mathrm{g} / \mathrm{ml}$ for all PMCA reactions. Prior to the amplification procedure, $19 \mu \mathrm{L}$ of the PMCA reaction mixture were taken (from the final volume of $120 \mu \mathrm{l}$ ) for each reaction (referred to as the "frozen" sample) for comparison with the sonicated samples (referred to as the "sonicated" sample). Amplification employed cycles of sonication and incubation in a programmable sonicator (Qsonica, model Q-700) at $37{ }^{\circ} \mathrm{C}$. A total of 96 PMCA cycles were performed comprising $20 \mathrm{~s}$ of sonication (at an amplitude of $38 \%$, wattage: 278-299) followed by 29 min $40 \mathrm{~s}$ of incubation for each cycle. Frozen and sonicated samples were digested with proteinase $\mathrm{K}$, and analysed by Western blotting using the monoclonal antibody 3F4 as described previously [1]. Amplification efficiency was expressed as the increase in $\mathrm{PrP}^{\mathrm{res}}$ signal in the sonicated compared to the frozen sample, as judged by densitometry of Western blot images acquired and analysed using the ChemiDoc ${ }^{\mathrm{TM}}$ XRS + System with Image Lab Software (Bio-Rad, Hemel Hempstead, UK).

\section{Statistics}

In the statistical analysis of the clinical patient information statistical significance was determined between the different PRNP codon 129 genotypes using a one-way ANOVA test followed by the Tukey's multiple comparisons test. Statistical analysis of all clinical data was performed using GraphPad Prism software 7.00. In the statistical analysis of RT-QuIC data, a two sided Mann-Whitney test was used to assess the statistical significance of differences between the median RT-QuIC lag periods of sCJD versus hGH-iCJD or hDM-iCJD cases. The Kruskal-Wallis test was used for the combined analysis of sCJD (VV2, MV2, MM1) and hGHiCJD (VV2, MV2) subgroups. These non-parametric tests were employed on the basis that the shapes of the distributions of the lag periods were similar for various subgroups. Statistical analysis and graphing of the data was performed using GraphPad Prism 7.00. 


\section{Results}

\section{$P R N P$ codon 129 genotype of the hGH-iCJD cases}

There have been 78 cases of hGH-iCJD identified in the UK from 1985 to the present day. 43 of these had fixed and/ or frozen tissue referred for diagnosis at the NCJDRSU. Of these 43 cases, 40 were autopsy cases and three were biopsy cases. 37 of these 43 cases had available codon 129 genotype data. 21 of the autopsy cases had frozen CNS tissues that were available for research use. The study identification numbers with clinical and pathological features of these 21 autopsy cases are shown in Online Resource Tables 1 and 2, respectively. When the hGH-iCJD patient $P R N P$ codon 129 status is considered by year of death the following becomes apparent: first, the 21 cases of hGHiCJD with frozen tissue analysed in this study died during the period from 1990 to 2012, and consequently these cases provide examples from the leading edge, the peak and the tail of the epidemic (Fig. 1a, b). Second, the UK hGH-iCJD group is dominated by patients of the MV and VV PRNP codon 129 genotypes; the few hGH-iCJD cases
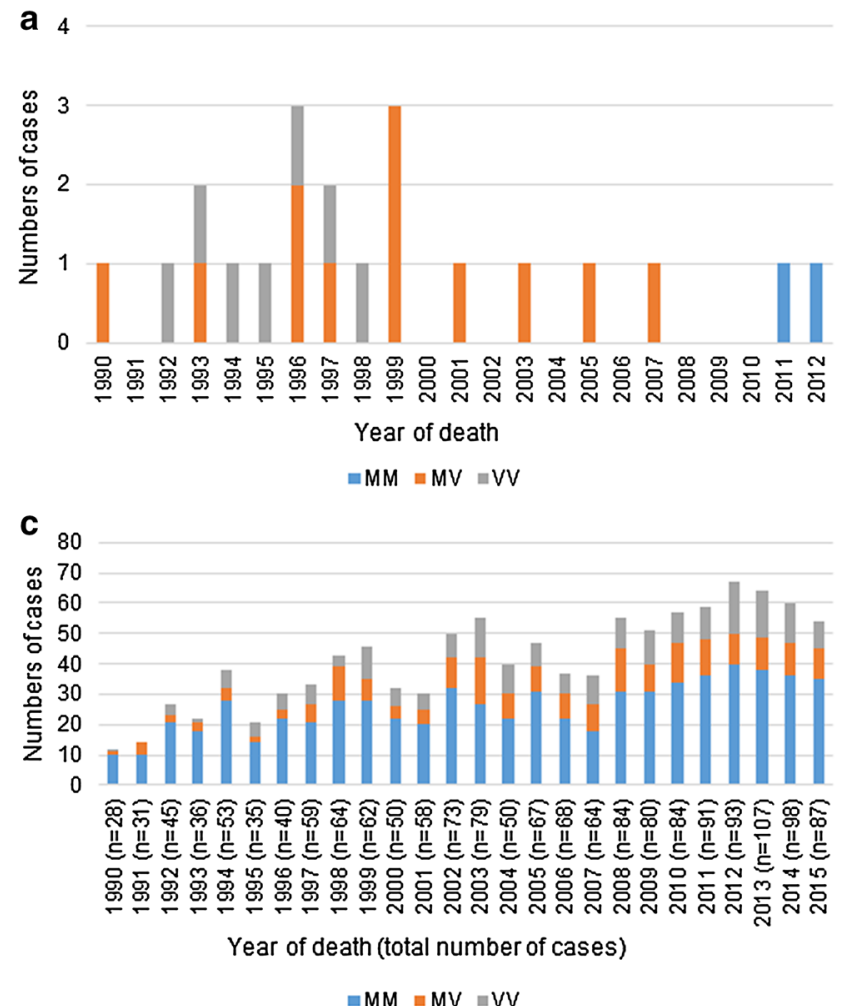

Fig. $1 P R N P$ codon 129 genotype of hGH-iCJD and sCJD patient groups. a Shows the PRNP codon 129 distribution of hGH-iCJD cases by year of death for the 21 cases investigated in this study. $\mathbf{b}$ Shows the PRNP codon 129 distribution of hGH-iCJD cases by year of death for the 37 cases for which PRNP codon 129 data is available to us. c Shows the PRNP codon 129 distribution by year of death that occurred in individuals with an MM PRNP codon 129 genotype tended to occur later than those of the MV and VV PRNP codon 129 genotypes (Fig. 1a, b). In contrast, the MM PRNP codon 129 genotype is the most frequently occurring group within the UK SCJD group overall, and in every year during the hGH-iCJD epidemic (Fig. 1c). Finally, when considered as a whole, the distribution of codon 129 genotypes of the 21 available hGH-iCJD cases and the 108 sCJD cases selected for study here differ from each other and from the normal UK population, but they appear to be broadly representative subsets of the larger hGH-iCJD and sCJD cohorts (Fig. 1d). The three hDMiCJD cases were all of the PRNP codon 129 MM genotype (Online Resource Table 2).

\section{hGH-iCJD patient information in relation to $P R N P$ codon 129 genotype group}

Age at death, duration of hGH treatment and estimated incubation periods were similar between the PRNP codon $129 \mathrm{MV}$ and VV genotype hGH-iCJD groups, but the incubation periods in the two MM cases were longer. In

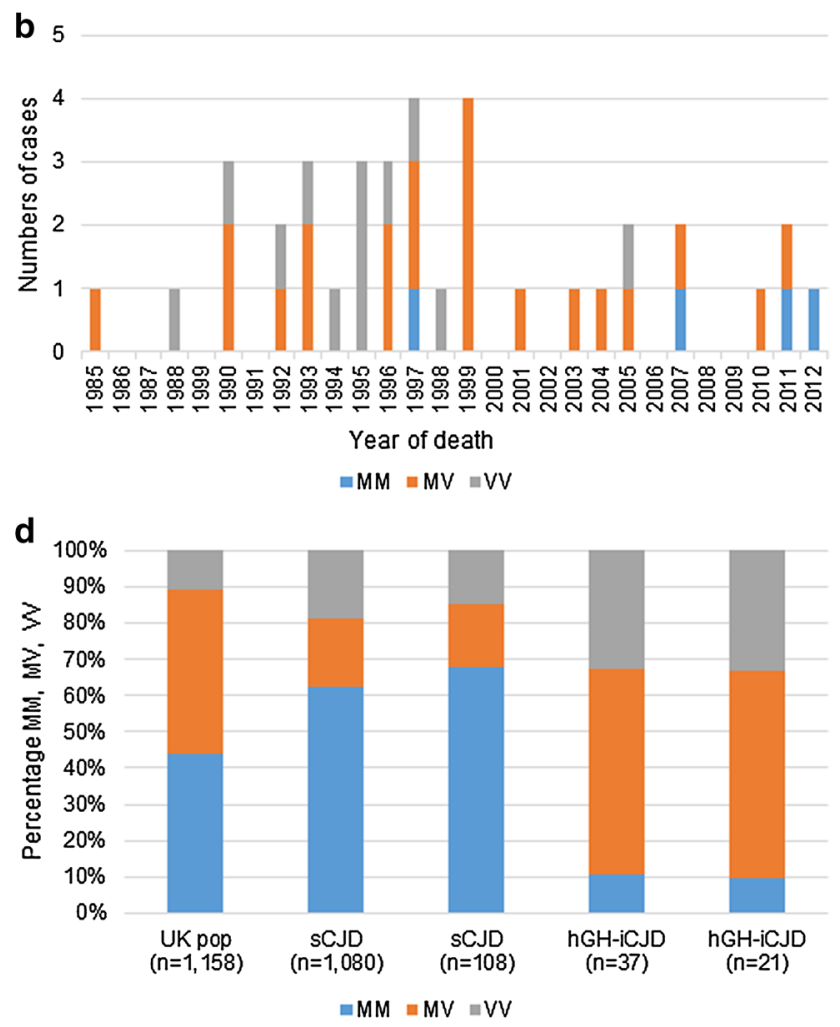

for the 1080 UK sCJD cases from 1990 to 2015. d Compares the PRNP codon 129 distribution of the hGH-iCJD $(n=21)$ and SCJD $(n=108)$ cases with frozen tissue analysed in this study, with the cases shown in $\mathbf{b}$ and $\mathbf{c}$, of which they are a subset. The UK normal population PRNP codon 129 genotype distribution (UK pop), is shown for reference 
contrast, the mean disease duration of illness of the MV group was longer than that of both the VV and MM groups (Online Resource Table 3).

\section{PrP ${ }^{r e s}$ types found in the hGH-iCJD cases}

Western blot analysis of all available CNS tissue from the 21 cases of hGH-iCJD for protease resistant prion protein $\left(\mathrm{PrP}^{\mathrm{res}}\right)$ was performed. Four $\mathrm{PrP}^{\mathrm{res}}$ types were identified in the hGH-iCJD cases, differing in the mobility and in the number of unglycosylated bands found. Representative examples of the $\operatorname{PrP}^{\text {res }}$ types $1, \mathrm{i}, \mathrm{i}+2$ and 2 in these cases are shown in Fig. 2a, example Western blots for each individual case are shown in Fig. 3, and the data compiled with the PRNP codon 129 genotype are shown in Online Resource Tables 1 and 2. The majority of cases were characterised by the presence of the $19 \mathrm{kDa}$ type 2 $\operatorname{PrP}^{\mathrm{res}}$ band (19/21 cases). However, in 12 out of these 19 cases the $20 \mathrm{kDa}$ type i $\mathrm{PrP}^{\mathrm{res}}$ band was also present. The relative abundance of the $19 \mathrm{kDa}$ type $2 \mathrm{PrP}^{\text {res }}$ band and the $20 \mathrm{kDa}$ type i $\operatorname{PrP}^{\mathrm{res}}$ band was variable, but the $19 \mathrm{kDa}$ type $2 \mathrm{PrP}^{\mathrm{res}}$ band usually predominated (Fig. 3a). Type i was found to predominate overall in only one hGH-iCJD case (hGH-iCJD20) and the $21 \mathrm{kDa}$ type $1 \mathrm{PrP}^{\mathrm{res}}$ was found in only one hGH-iCJD case (hGH-iCJD21). In contrast to the hGH-iCJD cases, all three cases of hDM-iCJD cases had type $1 \mathrm{PrP}^{\text {res }}$ exclusively (Fig. 3b). Where it could be assessed, regional variation within the brain was largely confined to the presence or absence of the $\operatorname{PrP}^{\text {res }}$ type $\mathrm{i}$ band and the relative abundance of $\operatorname{PrP}^{\text {res }}$ types $\mathrm{i}$ and 2 bands (Online Resource Table 4). Comparison of the immunoreactivity of the $21 \mathrm{kDa}$ type 1, $20 \mathrm{kDa}$ type i, and $19 \mathrm{kDa}$ type 2 bands using the 3F4 antibody (epitope 106112 ) with the $12 \mathrm{~B} 2$ antibody (epitope $89-93$ ) showed that the $21 \mathrm{kDa}$ type $1 \mathrm{PrP}^{\mathrm{res}}$ and the $20 \mathrm{kDa}$ type i PrP ${ }^{\mathrm{res}}$ fragments both retain the $12 \mathrm{~B} 2$ epitope, whereas type $2 \mathrm{PrP}^{\mathrm{res}}$
Fig. 2 PrP $^{\text {res }}$ type found in the iCJD cases examined. a Four $\operatorname{PrP}^{\text {res }}$ molecular types were detected by Western blot analysis of proteinase $\mathrm{K}$ treated brain extracts of hGH-iCJD patients; type 1 , type i, type $i+2$ and type 2 . Representative blots of each $\operatorname{PrP}^{\text {res }}$ type are shown. Each sample (middle lane) is flanked by type 1 (T1) (left lane) and type 2 (T2) (right lane) reference standards from sCJD MM1 and VV2 subtype cases, respectively. Case number and codon $129 P R N P$ genotype are indicated above each blot, whilst $\mathrm{PrP}^{\mathrm{res}}$ type is indicated below. The most informative exposure for the test and reference standard samples from a series of timed exposures is shown. Blots were probed using the monoclonal antibody 3F4. b Western blot analysis of proteinase $\mathrm{K}$ treated samples of $\mathrm{PrP}^{\mathrm{res}}$ types detected in hGHiCJD patients using the monoclonal antibody 12B2 which detects type $1 \mathrm{PrP}^{\mathrm{res}}$ and shown after a prolonged (30 $\mathrm{min})$ and abbreviated ( $3 \mathrm{~min}$ ) exposure time. Approximate molecular mass is shown in $\mathrm{kDa}$. The immunoblots shown are representative of at least three technical repeats
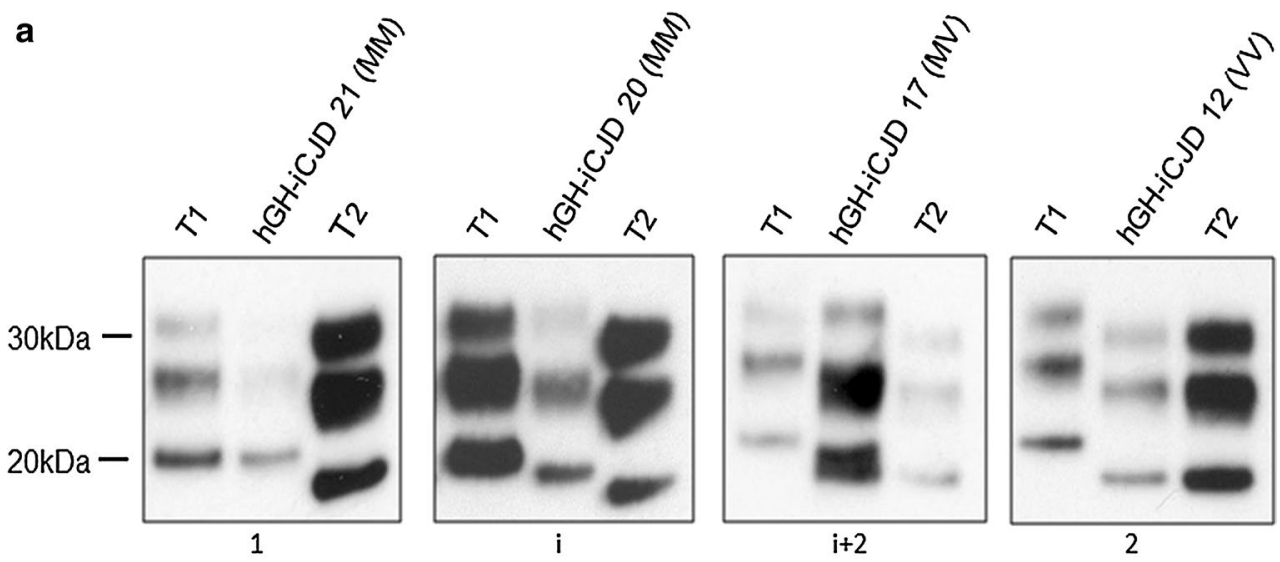

b

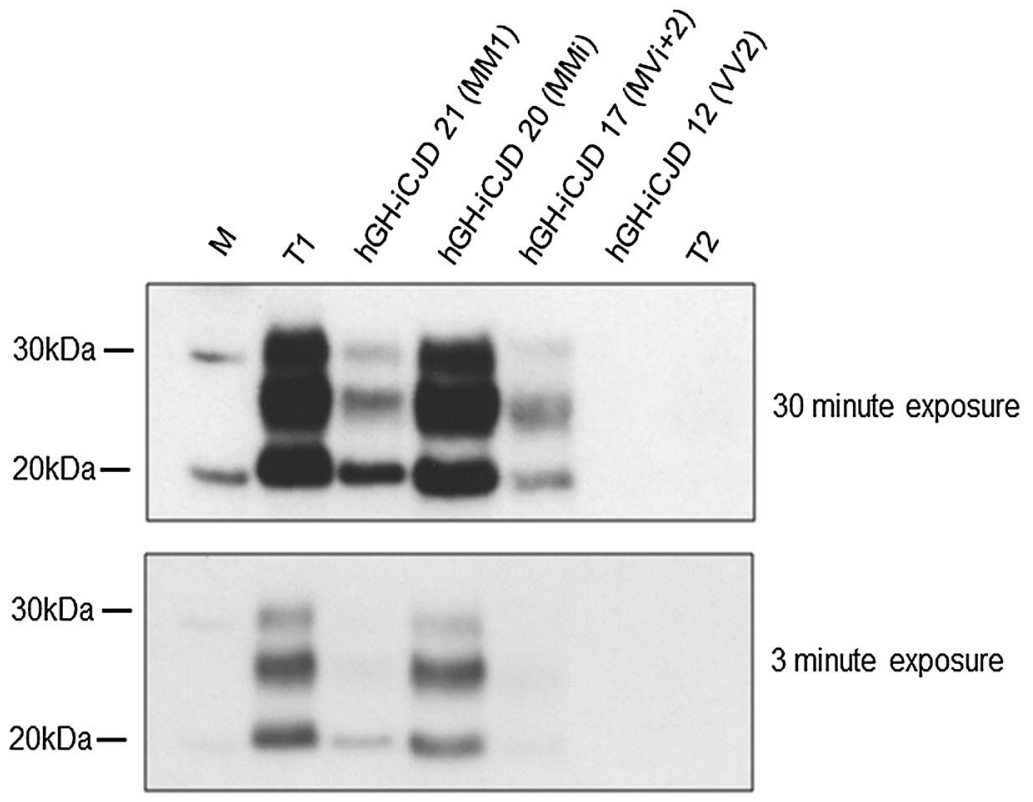


fragments do not (Fig. 2a, b). Analysis of the PrP ${ }^{\text {res }}$ types found in the CNS of 108 sCJD cases showed type 1, type 2 , type $i+2$ and cases in which type 1 and type 2 were found in individual brains, either in the same or in different regions (Online Resource Table 5). These latter cases were designated type $1+2$, irrespective of the relative abundance and location of the two types. The breakdown of these 108 SCJD cases according to the combination of CNS PrP ${ }^{\text {res }}$ type and PRNP codon 129 genotype is shown in Online Resource Table 6, while the histotypes and $\operatorname{Pr}^{\mathrm{res}}$ types associated with the PRNP codon $129 \mathrm{MV}$ sCJD cases are shown in Online Resource Table 7.

\section{PrPres type and PRNP codon 129 genotype of the hGH-iCJD cases}

When the 21 cases of hGH-iCJD were grouped according to the combination of PRNP genotype and CNS PrP ${ }^{\text {res }}$ type (Online Resource Table 2; Fig. 4a) all PRNP codon $129 \mathrm{VV}$ hGH-iCJD cases (7/7) were found to have type 2 PrP $^{\text {res }}$ exclusively. All of the hGH-iCJD PRNP codon 129 MV genotype cases were found to have type $\mathrm{i}+2 \operatorname{PrP}^{\text {res }}$ (12/12). Of the two PRNP codon 129 MM genotype cases found within the hGH-iCJD cohort, one had type $1 \mathrm{PrP}^{\text {res }}$ and the other had type i PrP ${ }^{\text {res }}$. In contrast, within the 108 sCJD cases, those with type $1 \mathrm{PrP}^{\mathrm{res}}$ and a PRNP codon 129 MM genotype predominated (50/108) and cases with type $1+2 \operatorname{PrP}^{\text {res }}$ were relatively common $(26 / 108)$, but no examples of type i $\mathrm{PrP}^{\mathrm{res}}$ alone were found (Online Resource Table 6; Fig. 4b). The three hDM-iCJD cases were in individuals of the PRNP codon 129 MM genotype and each had PrP ${ }^{\text {res }}$ type 1 in the CNS (Fig. 3b).

\section{Neuropathology of hGH-iCJD}

The neuropathological features were reviewed in the 21 hGH-iCJD patients (fixed tissue from case hGH-iCJD1 was not available for PrP immunohistochemistry). All cases showed a general widespread spongiform encephalopathy of a predominantly microvacuolar type accompanied by neuronal loss and gliosis, with amyloid plaques in the cerebellum in 13/21 cases. The patterns of accumulation of the disease-associated prion protein on immunohistochemistry were recorded in the 20 cases with available material. A summary of the neuropathological features of each hGH-iCJD case is added to the PRNP codon 129 genotype and $\mathrm{PrP}^{\mathrm{res}}$ type in Online Resource Table 2 and example micrographs are shown in Fig. 5 (the neuropathological features in this cohort will be described more fully in a separate manuscript). Overall, the patterns of pathology in the hGH-iCJD patients showed close similarities with sCJD patients of the corresponding PRNP codon 129 genotype.
Immunohistochemistry on tissue sections from PRNP codon $129 \mathrm{VV}$ hGH-iCJD patients showed a combination of synaptic, perineuronal and plaque-like deposits of PrP in the cerebral cortex often in a distinctive linear distribution in the deeper cortical layers, a pattern of PrP pathology very similar to that in the VV2 subtype of sporadic CJD (Fig. 5a, i). The cerebellum in these cases showed a coarser deposition of $\operatorname{PrP}$ with occasional plaque-like deposits (Fig. 5e, m). In contrast, all PRNP codon $129 \mathrm{MV}$ hGHiCJD patients were characterised by a pathology dominated by the accumulation of large and numerous kuru-type amyloid plaques in the cerebellar cortex and frequently in the cerebral cortex and basal ganglia, consistent with that observed in the sCJD MV2 subtype (Fig. 5b, f, j, n). The two hGH-iCJD PRNP codon $129 \mathrm{MM}$ cases available for analysis showed strikingly different patterns of neuropathology. In patient hGH-CJD20, the spongiform change comprised a mixture of both microvacuolar and confluent types, with PrP staining showing a combination of synaptic, perineuronal and perivacuolar PrP deposits (Fig. 5c). In addition, plaque-like deposits and kuru plaques were a prominent feature in the cerebral and cerebellar cortex from this case (Fig. 5g). In contrast, hGH-CJD21 showed spongiform change of predominantly the microvacuolar type with a widespread synaptic/granular pattern of PrP deposition, a pattern more consistent with that observed in the sCJD MM1 subtype (Fig. 5d, h, k, o). In the two hDMiCJD cases in which fixed tissue was available for immunohistochemical analysis, both showed a synaptic deposition of PrP throughout the brain. Kuru-type amyloid plaques or plaque-like $\mathrm{PrP}^{\text {res }}$ deposits were not observed in either case (Fig. 5l, p).

\section{Comparison of the amyloid seeding potential of hGH-iCJD and SCJD}

The amyloid seeding activity of hGH-iCJD and SCJD cases was compared using the real-time quaking induced conversion assay (RT-QuIC). hGH-iCJD cases were selected on the basis of the availability of frozen cerebral cortex samples $(n=20)$. sCJD cases $(n=28)$ were selected on the basis of $\mathrm{PrP}^{\text {res }}$ type and $P R N P$ codon 129 genotype to match, as far as possible, those found in the hGH-iCJD cohort. RT-QuIC assays were seeded with CJD cerebral cortex homogenate diluted by a factor of $2 \times 10^{-5}$ (each $100 \mu \mathrm{l}$ RT-QuIC reaction was seeded with the equivalent of $2 \times 10^{-7} \mathrm{~g}$ brain) and the seeding activity for each patient was assumed to be inversely proportional to the mean lag period (Table 1). The hGH-iCJD and sCJD seed amounts were determined empirically to be within the range for quantifying seeding activity by the lag time method. The hGH-iCJD group as a whole (and their subgroups) have a lower median seeding activity than the SCJD group (and their matched subgroups). The 

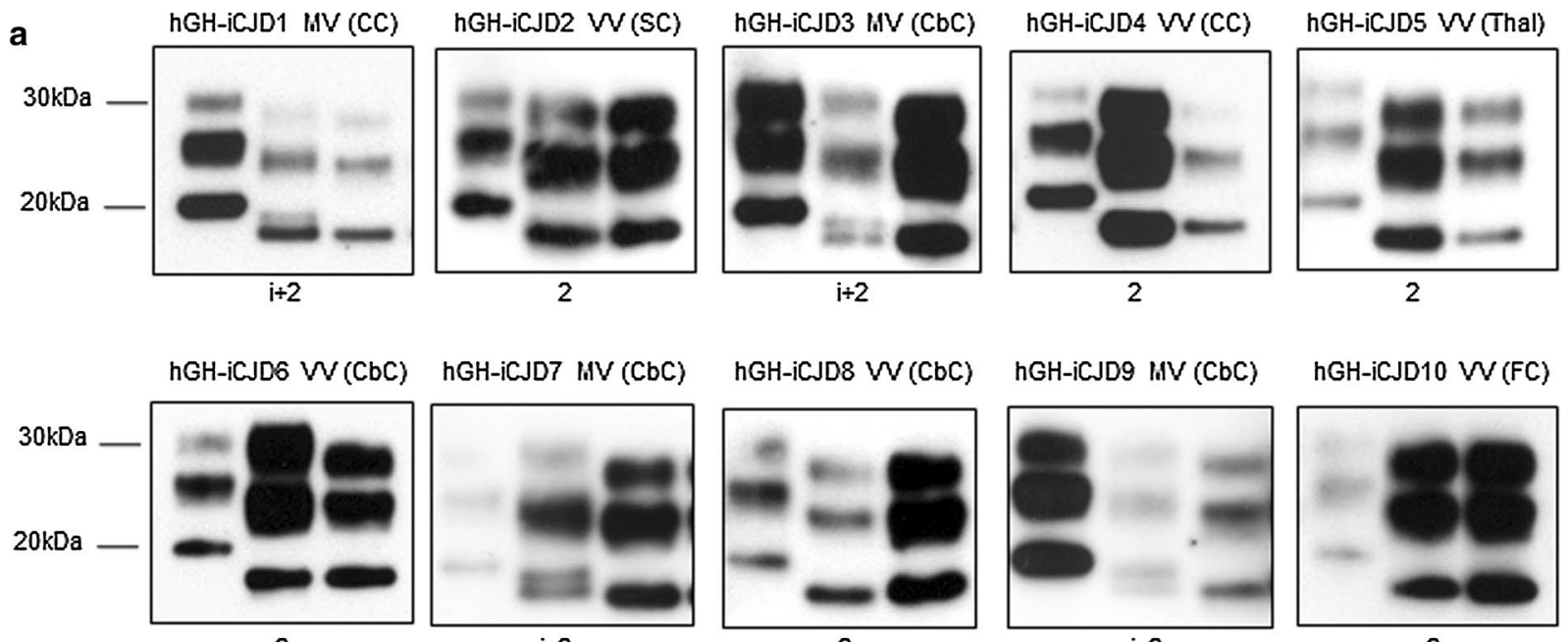

hGH-iCJD7 IV (CbC)

hGH-iCJD8 W(CbC)

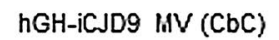

hGH-iCJD10 W(FC)

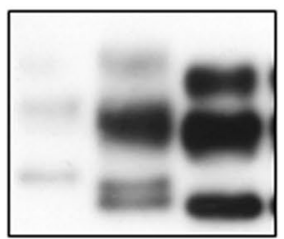

$\mathrm{i} \div 2$

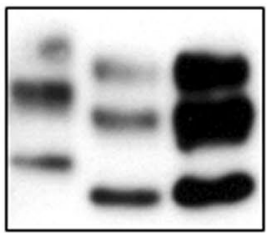

2

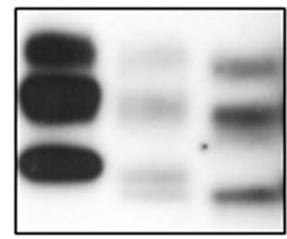

$\mathrm{i} \div 2$

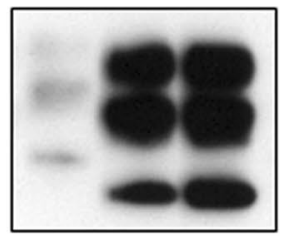

2
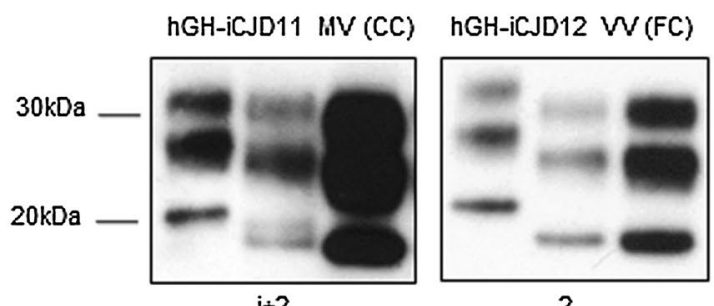

2

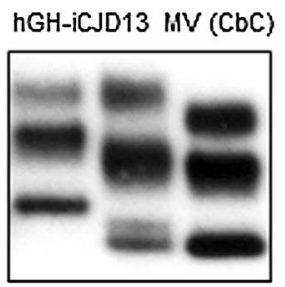

$\mathrm{i}+2$

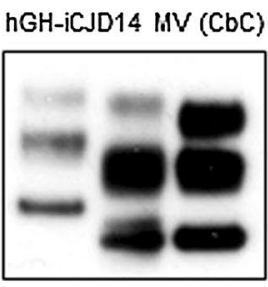

$\mathrm{i}+2$

hGH-iCJD15 INV (PC)

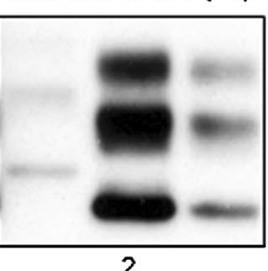

2
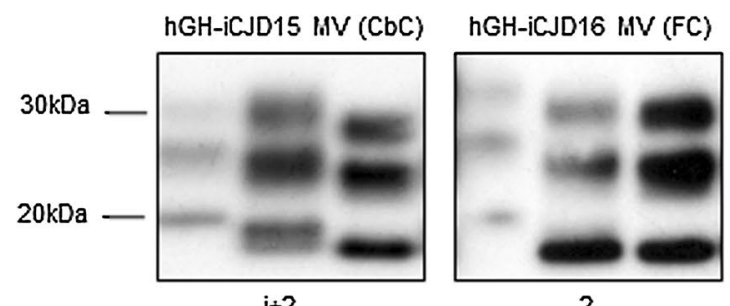

2

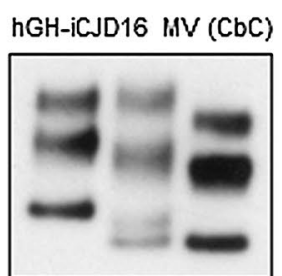

$\mathrm{i}+2$

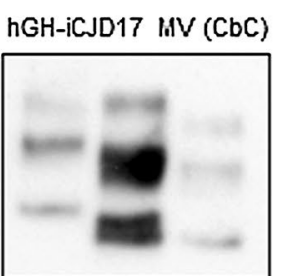

$i+2$
hGH-iCJD18 WV (CbC)

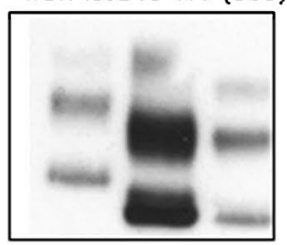

$\mathrm{i}+2$
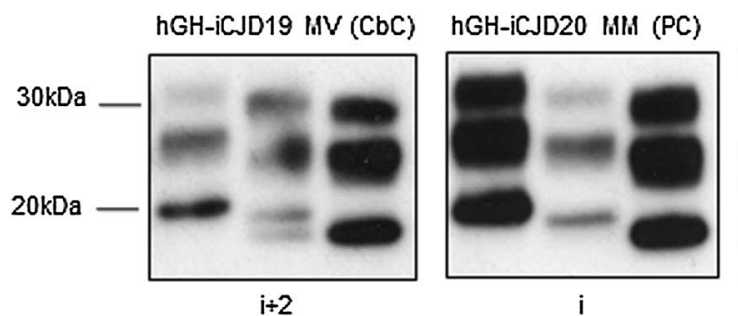

hGH-iCJD21 HA (CC)

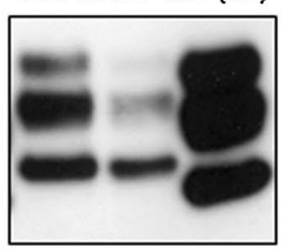

1

b

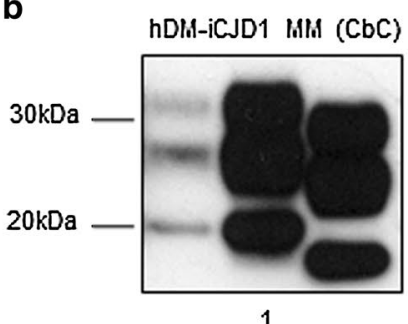

hDM-iCJD2 MM (FC)

hDM-iCJD3 MM (PC)
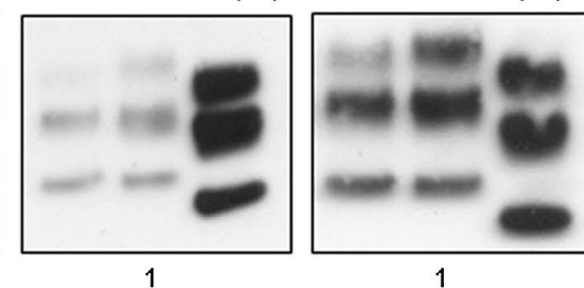
4Fig. 3 PrP $^{\text {res }}$ type found in each individual iCJD case examined. $\mathrm{PrP}^{\text {res }}$ typing of (a) hGH-iCJD cases $1-21$ and (b) hDM-iCJD cases 1-3. Each sample (middle lane) is flanked by a type 1 (left lane) and type 2 (right lane) reference standard from SCJD MM1 and VV2 subtype cases, respectively. Study identification number, PRNP codon 129 genotype and brain region are indicated above each blot and $\mathrm{PrP}^{\mathrm{res}}$ type is indicated below. Approximate molecular mass is shown in $\mathrm{kDa}$. The immunoblots shown are representative of at least three technical repeats

difference is significant for the hGH-iCJD group as a whole and for the VV and MV subgroups. The two PRNP codon $129 \mathrm{MM}$ hGH-iCJD cases had noticeably different lag times in this assay (hGH-iCJD20 at $43.5 \mathrm{~h}$ and hGH-iCJD21 at $27.5 \mathrm{~h}$ ). Three hDM-iCJD cases were also analysed by RTQuIC and their median lag period (34.2 h) was also greater than the sCJD MM1 subgroup, but this difference was not statistically significant. Although these experiments were conducted with recombinant hamster PrP, and therefore with a substrate containing methionine at the position equivalent to human codon 129, our previous experience with RT-QuIC indicates that it is less sensitive than PMCA to the biologically relevant effects of seed and substrate sequence matching or mismatching [26].

\section{Comparison of the in vitro amplification of hGH-iCJD and SCJD}

The seeding characteristics of the two divergent hGH-iCJD PRNP codon 129 MM cases (hGH-iCJD20 and hGHiCJD21) were further examined using protein misfolding cyclic amplification (PMCA). Samples of cerebral cortex were used to seed matched (PRNP codon 129 MM, designated HuMM) and mismatched (PRNP codon $129 \mathrm{VV}$, designated HuVV) PRNP transgenic mouse brain substrates and the results compared with those of SCJD subtypes. PMCA reactions seeded with cerebral cortex samples from cases of SCJD of the MM1 subtype amplified poorly under the conditions used, whether in matched (MM) or mismatched (VV) humanised transgenic mouse brain substrate (Fig. 6a). This was also true of the three hDM-iCJD cases (hDM-iCJD1-3) (Fig. 6a). Seeding of PMCA reactions with cerebral cortex samples from cases of sCJD of the VV2 subtype amplified more efficiently under these same conditions, with the amplification levels higher in the matched (HuVV) than in the mismatched (HuMM) humanised transgenic mouse brain substrate (Fig. 6a). The two hGH-iCJD PRNP codon $129 \mathrm{MM}$ cases gave divergent results: hGHiCJD20 amplified well and more efficiently in the HuVV substrate, whereas hGH-iCJD21 failed to seed either substrate efficiently (Fig. 6a). This difference in amplification efficiency and substrate preference between hGH-iCJD20 and hGH-iCJD21 was independent of seeding dilution (Fig. 6a). In the case of hGH-iCJD20, the choice of PRNP codon 129 substrate (HuMM or HuVV) also had an effect on the $\mathrm{PrP}^{\text {res }}$ type of the PMCA product when analysed by Western blotting (Fig. 6b). The cerebral cortex sample from hGH-iCJD20 was PrP $^{\text {res }}$ type i, but matching of the MMi seed with the HuMM substrate resulted in an upshift in mobility of the reaction product, whereas mismatching the MMi seed with the HuVV substrate resulted in an apparent downshift in mobility of the reaction product. hGH-iCJD21 amplified only poorly, and there was no evidence of any change in the type $1 \mathrm{PrP}^{\mathrm{res}}$ when the PMCA products were analysed by Western blotting (Fig. 6b). Addition examples of this reproducible, albeit subtle, mobility shift are shown (Online Resource Fig. 1). The possibility that $\operatorname{PrP}^{\text {res }}$ type $\mathrm{i}$ results from replication of the $\mathrm{SCJD}$ with type $2 \mathrm{PrP}^{\mathrm{res}}$ in an individual with a PRNP codon $129 \mathrm{MM}$ genotype was then modelled using PMCA. When sCJD VV2 subtype brain homogenate was used to seed the HuMM substrate, an upward shift was seen, whereas amplification of the same seed in a PRNP codon $129 \mathrm{VV}$ genotype resulted in conservation of the type $2 \operatorname{PrP}^{\text {res }}$ (Fig. 6c). Further examples of this phenomenon using four different cases of sCJD VV2 subtype are shown (Online Resource Fig. 2).

\section{Comparison of the two hGH-iCJD PRNP codon 129 MM cases}

PRNP codon $129 \mathrm{MM}$ is the rarest genotype within the UK $\mathrm{hGH}-\mathrm{iCJD}$ cohort and cases with this genotype have tended to occur late during the epidemic. The two hGH-iCJD PRNP codon $129 \mathrm{MM}$ cases available to this study were divergent in their neuropathology, $\operatorname{PrP}^{\text {res }}$ type and amplification potential in both the RT-QuIC and PMCA assays. In each of these respects, hGH-iCJD21 resembled the sCJD MM1 subtype (and the limited number of hDM-iCJD cases analysed), whereas hGH-iCJD20 more closely resembled the sCJD MV2 or VV2 subtypes, despite its PRNP codon $129 \mathrm{MM}$ genotype. These data are summarised in Table 2 and Fig. 7.

\section{Discussion}

This study provides a thorough molecular genetic description of the largest series of UK hGH-iCJD epidemic published to date, including as it does a large number of cases (21 compared to seven in a recent study [31]) in which the pathology, biochemistry and genetics can be compared. It also provides a description of the leading edge, peak and tail of the epidemic, rather than focussing largely on the tail of the epidemic [31]. In doing so, this study provides a unique opportunity to investigate the molecular epidemiology of prion transmission within a species, but across PRNP codon 129 polymorphic barriers. 


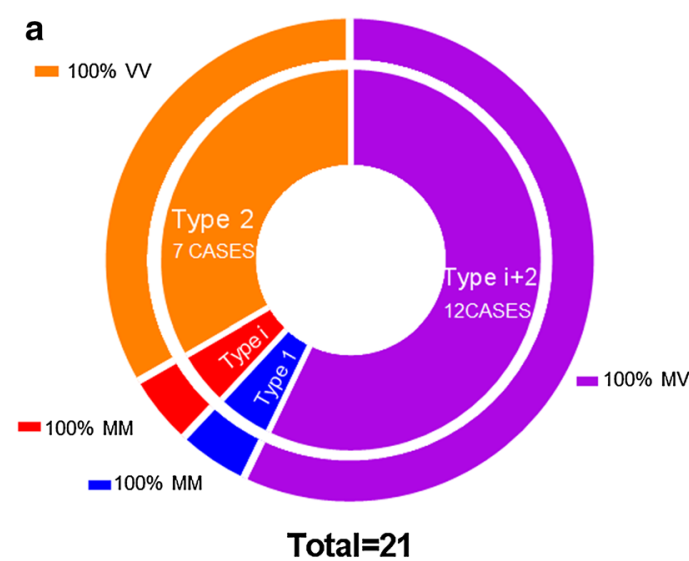

hGH-iCJD PRNP codon 129 genotype and PrPres type

Fig. 4 PrP $^{\text {res }}$ type and PRNP codon 129 genotype of the hGH-iCJD and SCJD groups. a Shows the numbers of cases found within the hGH-iCJD group $(n=21)$ according to the combination of $\operatorname{PrP}^{\text {res }}$ type (inner ring) and PRNP codon 129 genotype (outer ring). b

The UK hGH-iCJD epidemic is widely thought to result from contamination of hGH batches with prions present in tissue (either pituitary or brain) collected from one, or more cases of "classical" Creutzfeldt-Jakob disease. On the balance of probabilities, this is more likely to be a form of SCJD (rather than a genetic form) since sCJD accounts for $\sim 85 \%$ of human prion disease cases [12]. Although it cannot be known with certainty, the thesis proposed here is that the epidemiology of the epidemic is consistent with contamination with a single major prion strain.

Prion transmission (susceptibility, incubation period and phenotype) can be considered as an interaction between agent strain and host genotype, mediated in part by the related properties of abnormal prion protein conformation (for which $\operatorname{PrP}^{\text {res }}$ type is a surrogate marker) and the PRNP polymorphism that the encodes the residue at position 129 of the prion protein (either $\mathrm{M}$ or $\mathrm{V}$ ). At present, five sCJD prion strains (designated M1, M2(c), M2(t), V1 and V2) have been distinguished by transmission studies in PRNP humanised transgenic mice and nonhuman primates and the V2 strain has been proposed to account for both MV2 and VV2 sCJD [3, 21, 24]. The surfeit of PRNP codon 129 MM hGH-iCJD cases in France, compared to the predominance of $P R N P$ codon $129 \mathrm{VV}$ hGH-iCJD cases in the UK as of 2003 was suggested to be attributable to different contamination events by different strains of agent [4]. Given that the two most frequently occurring subtypes of sCJD are the MM1 and VV2 subtype, the simplest hypothesis is that the French hGH-iCJD epidemic was caused primarily by contamination with MM1 SCJD (i.e. the M1 SCJD strain) and the UK hGH-iCJD epidemic caused primarily by VV2 or MV2 sCJD contamination (i.e. the V2 sCJD strain).

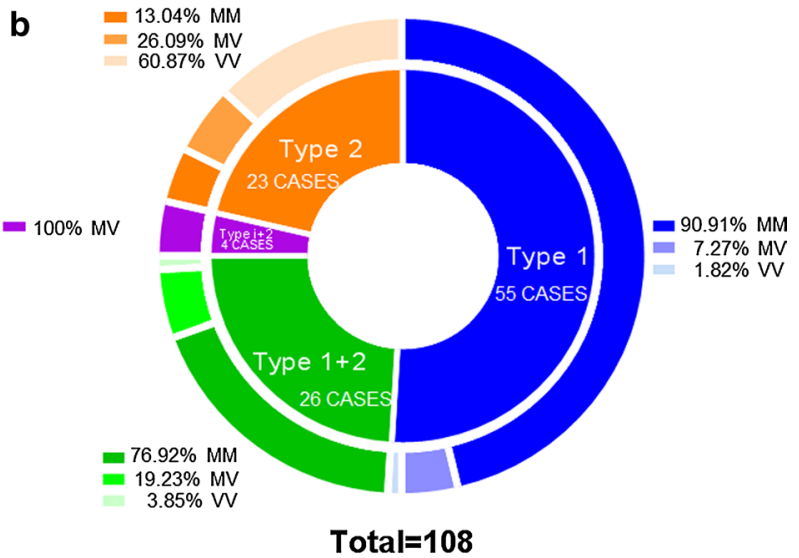

SCJD PRNP codon 129 genotype and PrPres type

Shows the numbers of cases found within the SCJD group $(n=108)$ according to the combination of $\mathrm{PrP}^{\mathrm{res}}$ type (inner ring) and PRNP codon 129 genotype (outer ring)

The data presented here and those of Rudge et al. [31] are consistent with that explanation, coupled with the PRNP genotype frequency of the at-risk hGH treated patient group, which if similar to UK control (e.g. blood donor) groups would be $44 \% \mathrm{MM}, 45 \% \mathrm{MV}$ and $11 \% \mathrm{VV}$ [2]. Hence, the early surfeit of PRNP codon $129 \mathrm{VV}$ UK hGH-iCJD cases could reflect the absence of a genotypic barrier and shorter incubation periods, albeit in a small subset of the hGH treated patient group. The appearance of more numerous cases in PRNP codon $129 \mathrm{MV}$ individuals may reflect the larger numbers of $P R N P$ codon $129 \mathrm{MV}$ individuals in the hGH treated patient group. The appearance of a limited number of PRNP codon $129 \mathrm{MM}$ hGH-iCJD cases, comparatively late in the epidemic, even although the hGH treated patient group would be expected to be $\sim 40 \%$, could be interpreted as evidence of a substantial PRNP codon 129 genotypic barrier to transmission of SCJD of the VV2 or MV2 subtypes, or a very prolonged incubation period. Although we cannot completely discount a mixture of contaminating strains including M1 in the form of sCJD MM1/MV1 subtype, the late appearance of these MM cases argues against a second minor M1 contaminating strain.

Our comprehensive data set coupled with the use of in vitro molecular conversion systems can be used to refine that model and provides a possible molecular mechanism for cross-genotype transmission. Within SCJD cases with PRNP codon $129 \mathrm{MV}$ and $\mathrm{PrP}^{\text {res }}$ type 2, some cases have kuru plaques (the MV2K subtype), whereas others do not (the MV2C subtype). The MV2K sCJD subtype is characterised by $\operatorname{PrP}^{\text {res }}$ type $\mathrm{i}+2$ (a doublet of approximately $20 \mathrm{kDa}$ and $19 \mathrm{kDa}$ ), whereas the MV2C subtype is reported to be characterised by the $19 \mathrm{kDa}$ band only, and 

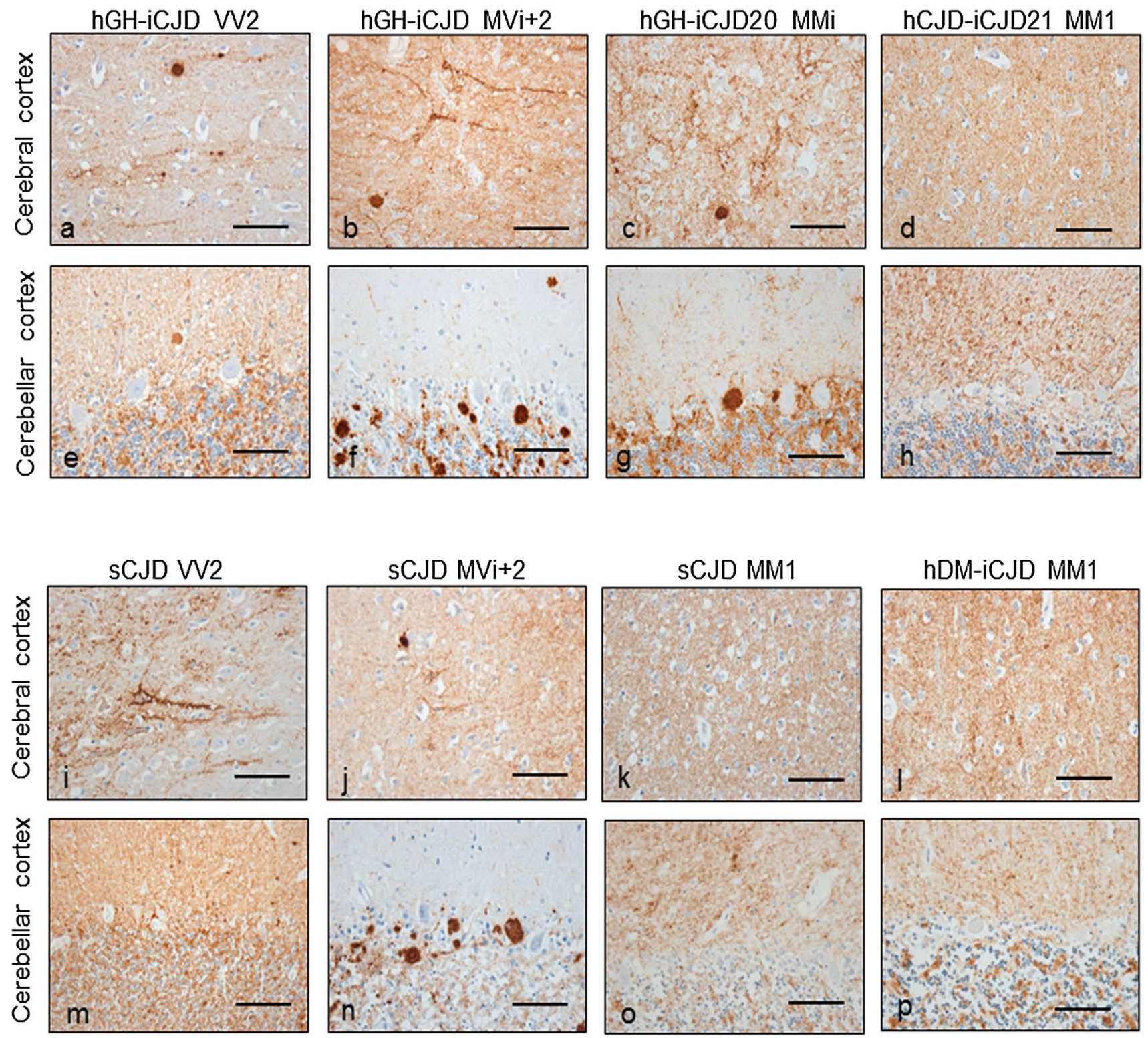

Fig. 5 Characteristic neuropathology of the SCJD and iCJD cases. Prominent patterns of PrP pathology in the cerebral and cerebellar cortex in UK hGH-iCJD (a-h), sCJD (i-k, m-o) and hDM-iCJD patients $(\mathbf{l}, \mathbf{p})$. PRNP codon $129 \mathrm{VV}$ hGH-iCJD patients showed a combination of synaptic, perineuronal and plaque-like deposits of $\operatorname{PrP}$ in the cerebral and cerebellar cortex (a, e). Intensely labelled kuru plaques and smaller plaque-like deposits were prominent features of the cerebral and cerebellar cortex in PRNP codon $129 \mathrm{MV}$ hGH-iCJD patients $(\mathbf{b}, \mathbf{f})$. Contrasting patterns of PrP pathology were observed in the two PRNP codon $129 \mathrm{MM}$ hGH-iCJD patients. Patient hGHiCJD20 showed plaque-like deposits in the cerebral cortex (c) and numerous kuru plaques in the cerebellum (g) whereas hGH-iCJD21

termed type $2[17,23,24]$. We note that all PRNP codon 129 MV UK hGH-iCJD cases had $\operatorname{PrP}^{\text {res }}$ type i + $2(12 / 12)$ and kuru plaques. One of the two PRNP codon $129 \mathrm{MM}$ hGH-iCJD cases described (hGH-iCJD20) also had kuru plaques (not normally found in PRNP codon $129 \mathrm{MM}$ showed only synaptic PrP deposits (d, h). The VV2 subtype in SCJD shows a perineuronal and synaptic pattern of PrP deposits in the cerebral and cerebellar cortex $(\mathbf{i}, \mathbf{m})$. The MV2 subtype of sCJD shows a synaptic and perineuronal deposition of $\operatorname{PrP}$ in the cerebral cortex (j) with kuru plaques and plaque-like deposits of PrP a dominant feature in the cerebellum (n). A widespread synaptic pattern of PrP accumulation is the characteristic feature of the MM1 subtype of SCJD (k, o). The two UK hDM-iCJD patients were also characterised by a predominantly synaptic pattern of PrP accumulation in the cerebral and cerebellar cortex $(\mathbf{l}, \mathbf{p})$. The anti-PrP antibody KG9 was used. Scale bars $50 \mu \mathrm{m}$

sCJD cases) and an intermediate $\sim 20 \mathrm{kDa} \operatorname{PrP}^{\text {res }}$ type (type i), perhaps suggestive of a causal link to the MV2K subtype of SCJD. Moreover, the in vitro amplification characteristics of $\mathrm{PrP}^{\mathrm{res}}$ from this case when used to seed the cell free prion protein conversion assay PMCA were not 


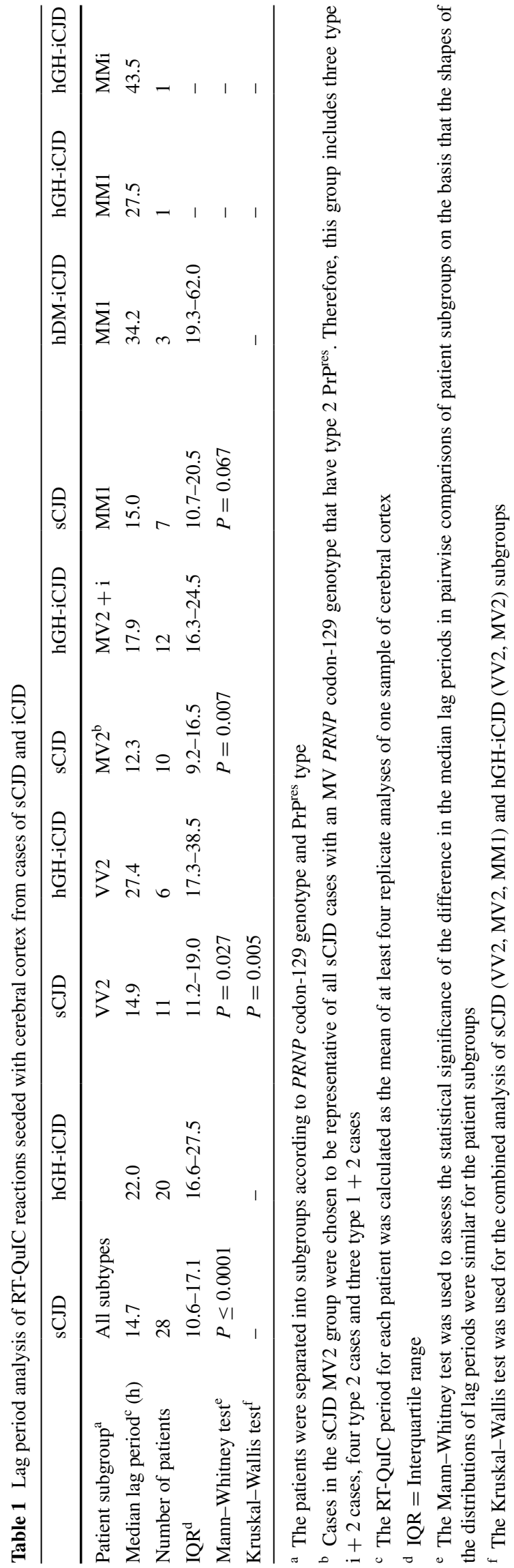

consistent with its PRNP codon $129 \mathrm{MM}$ genotype, instead showing a preference for a substrate containing $\operatorname{PrP}^{\mathrm{C}}$ with valine rather than methionine at position 129 . This situation is highly reminiscent of the molecular trace-back phenomenon proposed by Kitamoto and coworkers to explain the phenotypic diversity of the MV2 sCJD cases, and the transmission properties and origin of dura mater-associated CJD in Japan [15, 16, 18]. Furthermore, Kobayashi et al. [19, 20] have proposed that kuru plaques in PRNP codon 129 $\mathrm{MM}$ apparent SCJD cases can be used as an indication of an iatrogenic rather than idiopathic/sporadic aetiology. The characterisation of our case hGH-iCJD20 here extends the trace-back phenomenon of Kitamoto and coworkers from dura mater-associated transmissions in Japan to UK growth hormone associated transmissions. One of our PRNP codon $129 \mathrm{MM}$ hGH-iCJD cases has some similarities to one case recently described in a group of three hGH-iCJD cases in the USA [6]. The association of a PRNP codon $129 \mathrm{MM}$ genotype with kuru plaques in three national independent iCJD cohorts adds weight to the trace-back hypothesis. Moreover, our data using PMCA also confirms that evidence of the trace-back phenomenon in hGH-iCJD can be acquired from cell free systems, in addition to animal transmission studies, as predicted for hDM-iCJD by Kobayashi et al. [20] and subsequently demonstrated by Takeuchi et al. [34].

The molecular mechanism of the trace-back phenomenon is not fully understood, but the data of Kitamoto and coworkers points to a key role for the intermediate $\mathrm{PrP}^{\text {res }}$ type, termed type i. In this light, it is also tempting to suggest that our data points to the sCJD MV2K subtype as the source of the UK hGH-iCJD epidemic. It is plausible that the combination of the $20 \mathrm{kDa}$ type $\mathrm{i}$ and $19 \mathrm{kDa}$ type 2 $\mathrm{PrP}^{\mathrm{res}}$ conformational variants found in $\mathrm{SCJD} \mathrm{MV} 2 \mathrm{~K}$ might engender enhanced genotypic host range according to the principles of the conformational selection model proposed by Collinge and Clarke [7]. In this scheme, the MV2K subtype would transmit efficiently to MV individuals resulting in hGH-iCJD with a recognisable MV2K phenotype involving kuru plaques. The presence of type $2 \mathrm{PrP}^{\mathrm{res}}$ in the type $\mathrm{i}+2$ isoform would also facilitate efficient transmission to PRNP codon $129 \mathrm{VV}$ individuals and result in the recognisable VV2 phenotype lacking kuru plaques. Finally, the coexistence of type $\mathrm{i}$ with type $2 \mathrm{PrP}^{\mathrm{res}}$ within the type $2 \mathrm{~d}$ or 2 doublet isoform might render MV2K more compatible with transmission to PRNP codon $129 \mathrm{MM}$ individuals, but only with extended incubation periods and producing a phenotype with $\operatorname{PrP}^{\text {res }}$ type $\mathrm{i}$ and kuru plaques or, if molecular adaptation is complete, type $1 \operatorname{PrP}^{\mathrm{res}}$ and an MM1 phenotype.

Alternatively, a sCJD VV2 source of infection in the hGH-iCJD cohort would involve a molecular strain adaptation-based model (rather than a molecular strain 


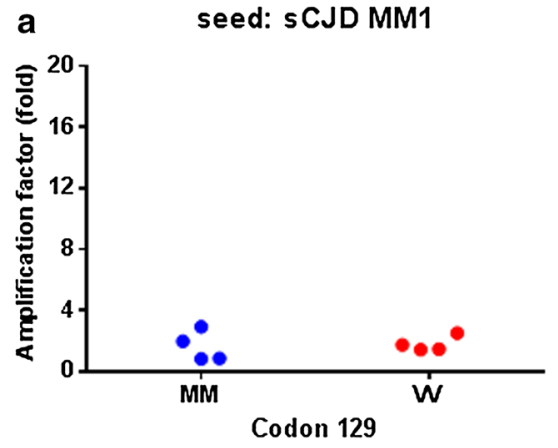

seed: hDM-iCJD MM1

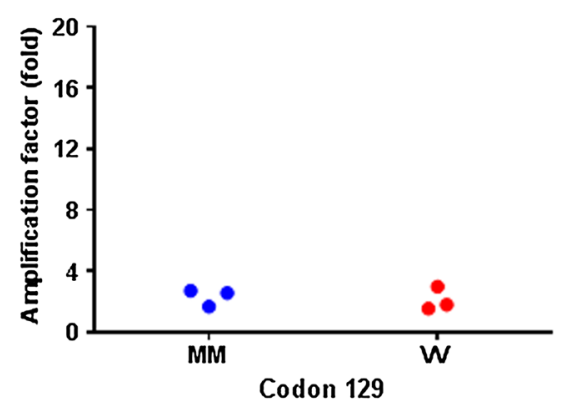

seed: hGH-iCJD MM

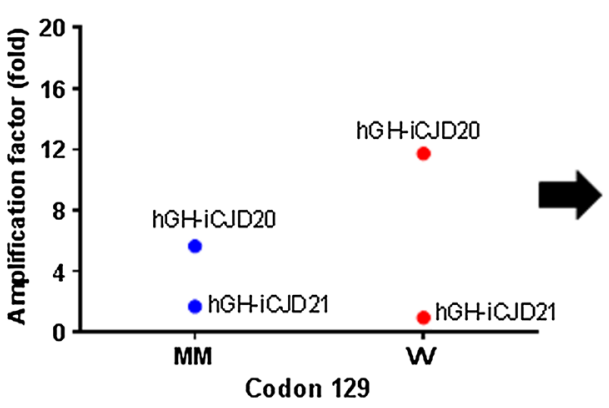

seed: sCJD MV2

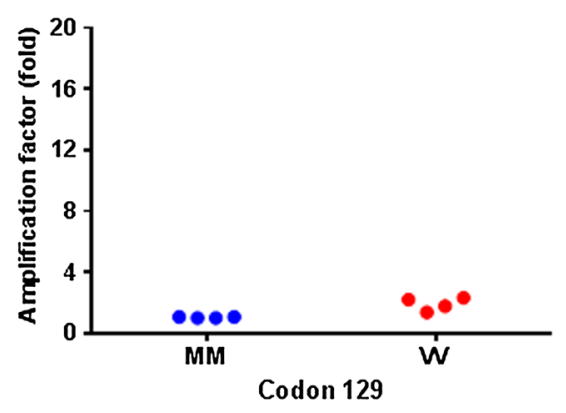

seed: hGH-iCJD MV2

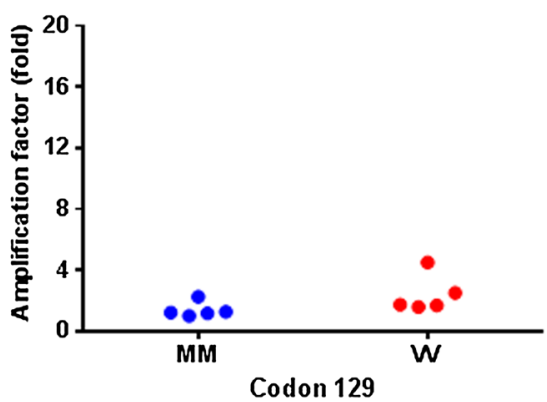

Substrate:

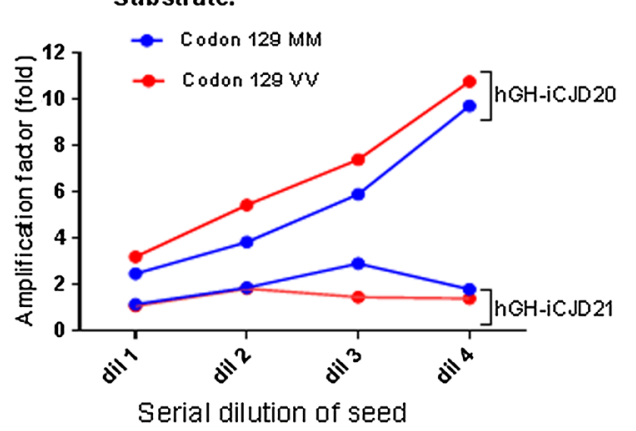

seed: sCJD VV2

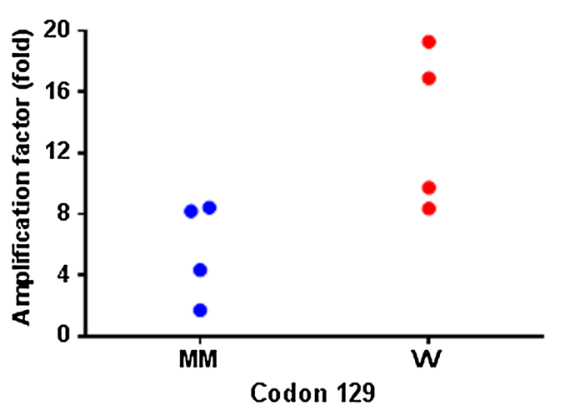

seed: hGH-iCJD VV2

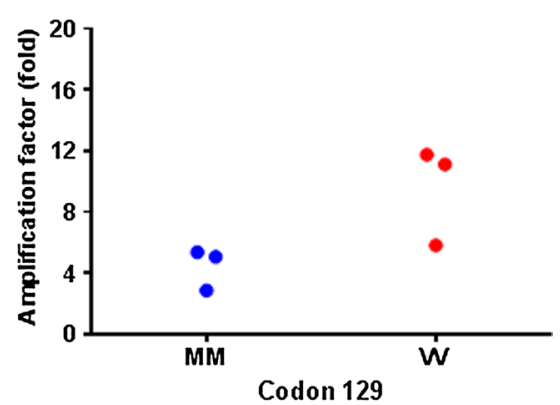

Fig. $6 \mathrm{PrP}^{\mathrm{res}}$ analysis of PMCA reactions seeded with cerebral cortex from case of sCJD and iCJD. a hGH-iCJD, hDM-iCJD and sCJD brain homogenate (seeds) were diluted in humanised transgenic mouse brain homogenate (substrate) of the PRNP codon 129 MM (red dots) or VV (blue dots). Amplification factor is shown as the relative increase (fold) in $\mathrm{PrP}^{\mathrm{res}}$ signal after a single round of PMCA as determined by densitometry of the Western blotting signal. PMCA reactions were normalized by seed $\operatorname{PrP}^{\text {res }}$ amount. PMCA of serial dilution of the hGH-iCJD MM seeds were also performed (hGH-iCJD20: 1/50, 1/100, 1/200, 1/400 and hGH-iCJD21: 1/4, 1/8, $1 / 16,1 / 32)$. PMCA experiments seeded with hGH-iCJD, hDM-iCJD and SCJD samples were performed twice independently and repeated at least four times for the reactions seeded with the hGH-iCJD MM cases. b PrP ${ }^{\text {res }}$ type of MM hGH-iCJD cases 20 and 21 assessed before and after PMCA. PMCA reactions employed matched (MM) and mismatched (VV) PRNP transgenic mouse brain substrates. Each sample was run between a type 1 (left lane) and type 2 (right lane) reference standards from SCJD MM1 and VV2 subtype cases, respectively (T1 and T2). Study identification numbers of the hGHiCJD MM cases are indicated. $\mathbf{c} \mathrm{PrP}^{\text {res }}$ analysis of sCJD VV2 PMCA products detected by Western blot. SCJD VV2 brain homogenate was diluted and amplified in matched (MM) and mismatched (VV) codon 129 substrate by a single round of PMCA. Samples corresponding to unamplified (Frozen) and amplified (PMCA) aliquots are shown with reference standards as described above. A total of 25 biological replicates of hGH-iCJD, hDM-iCJD and SCJD were analysed

Fig. 7b. The most relevant published humanised transgenic mouse model transmission data do not help in resolving the VV2/MV2 question, because the VV2 sCJD and MV2 sCJD cases used transmitted with indistinguishable characteristics in these mice. However, it is noteworthy that the most efficient transmission with the shortest incubation period was with the V2 strain (both VV2 and MV2 sCJD) in PRNP codon $129 \mathrm{VV}$ mice [3]. Distinguishing between is plotted according to estimated incubation periods in 


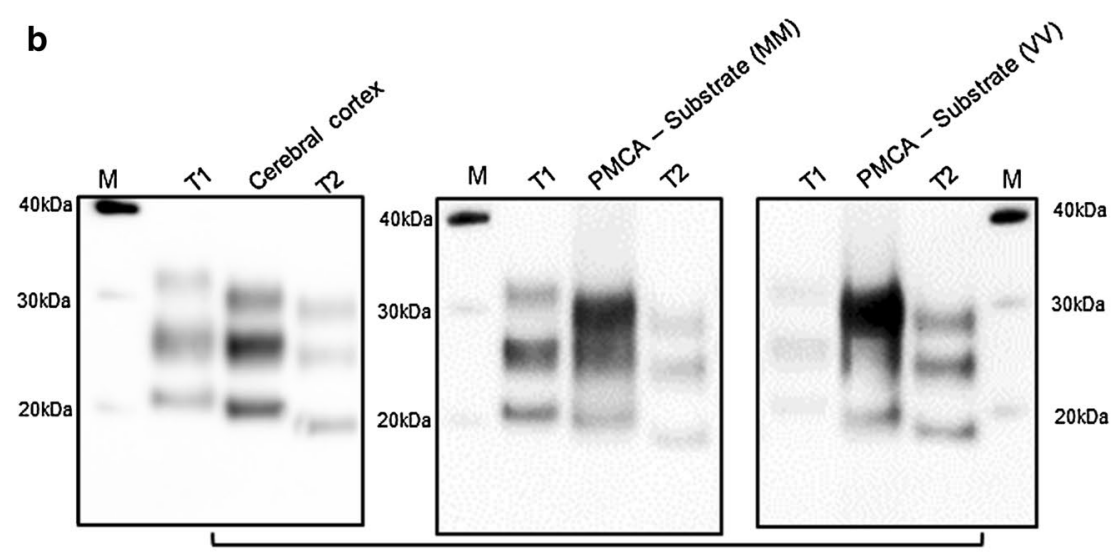

c

hGH-iCJD20

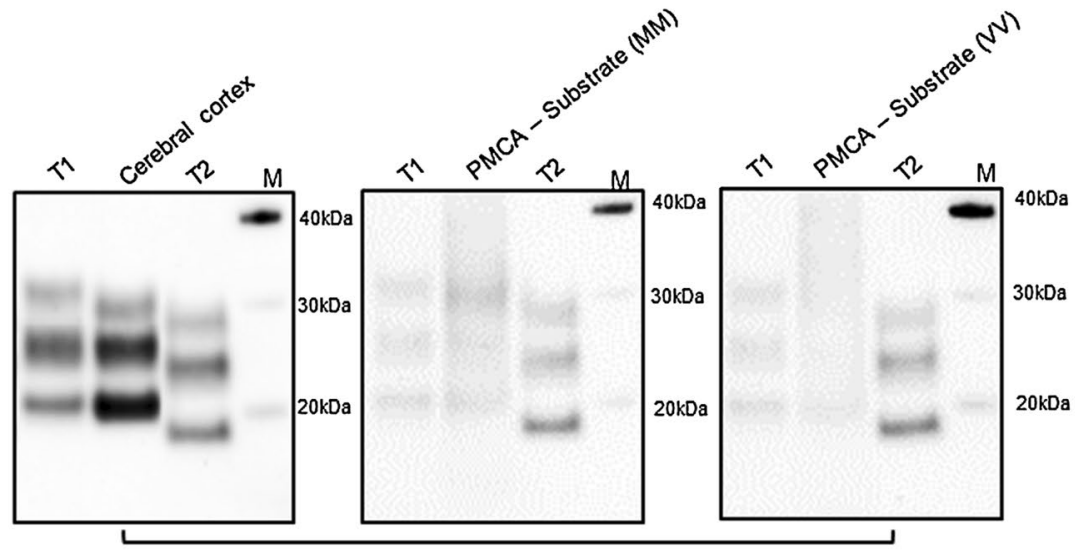

hGH-iCJD21

Fig. 6 continued

Table 2 Comparison of two hGH-iCJD (MM) cases with differing pathological and molecular features

\begin{tabular}{lll}
\hline & hGH-iCJD21 & hGH-iCJD20 \\
\hline PRNP codon 129 genotype & MM & MM \\
Neuropathology & Diffuse PrP positivity (resembling MM1 & PrP positive kuru plaques present \\
& sCJD and hDM-iCJD) & (similar to MV2K sCJD) \\
RT-QuIC seeding activity & Shorter lag time (closer to MM1 sCJD) & Long lag time \\
PrP & Type $1(\sim 21 \mathrm{kDa})$ & Type i ( 20 kDa) \\
PMCA efficiency & Poor & Greater \\
PMCA substrate preference & None & VV $>$ MM \\
PMCA product PrP ${ }^{\text {res }}$ mobility & Type 1 & MM Substrate Results In Upshift \\
& & VV Substrate Results In Downshift
\end{tabular}

sCJD VV2 and MV2K as the source of UK hGH-iCJD may be more than a formal difficulty given our recent finding that the proportion of $\mathrm{PrP}^{\text {res }}$ derived from the two $P R N P \mathrm{M}$ and $\mathrm{V}$ alleles can vary dramatically in SCJD, such that some heterozygous SCJD cases might in effect contain only $\mathrm{PrP}^{\mathrm{res}}$ derived from the $129 \mathrm{~V}$ or the $129 \mathrm{M}$ alleles [22]. Irrespective of mechanisms, the chronological

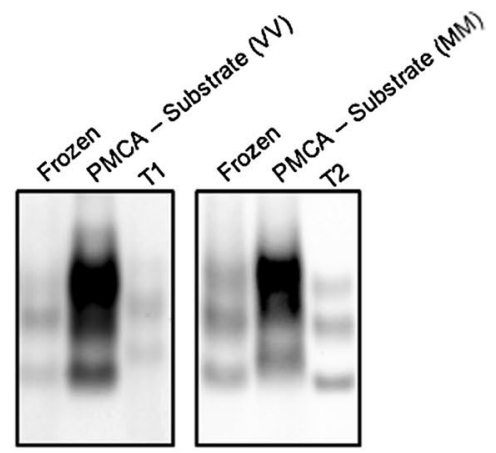

PMCA-seed: sCJD VV2 
Fig. 7 The UK hGH-iCJD epidemic and the study outcomes. a A summary of the molecular genetic finding of the hGH-iCJD cohort studied here showing correlations between PRNP codon 129 genotype, $\operatorname{PrP}^{\mathrm{res}}$ type and pathology, the genetics of the exposed group and the suspected source of the hGH-iCJD infectivity. b Graphic representation of the numbers of hGH-iCJD subtypes (PRNP codon 129 genotype and $\mathrm{PrP}^{\mathrm{res}}$ type) plotted according to increasing estimated incubation period a

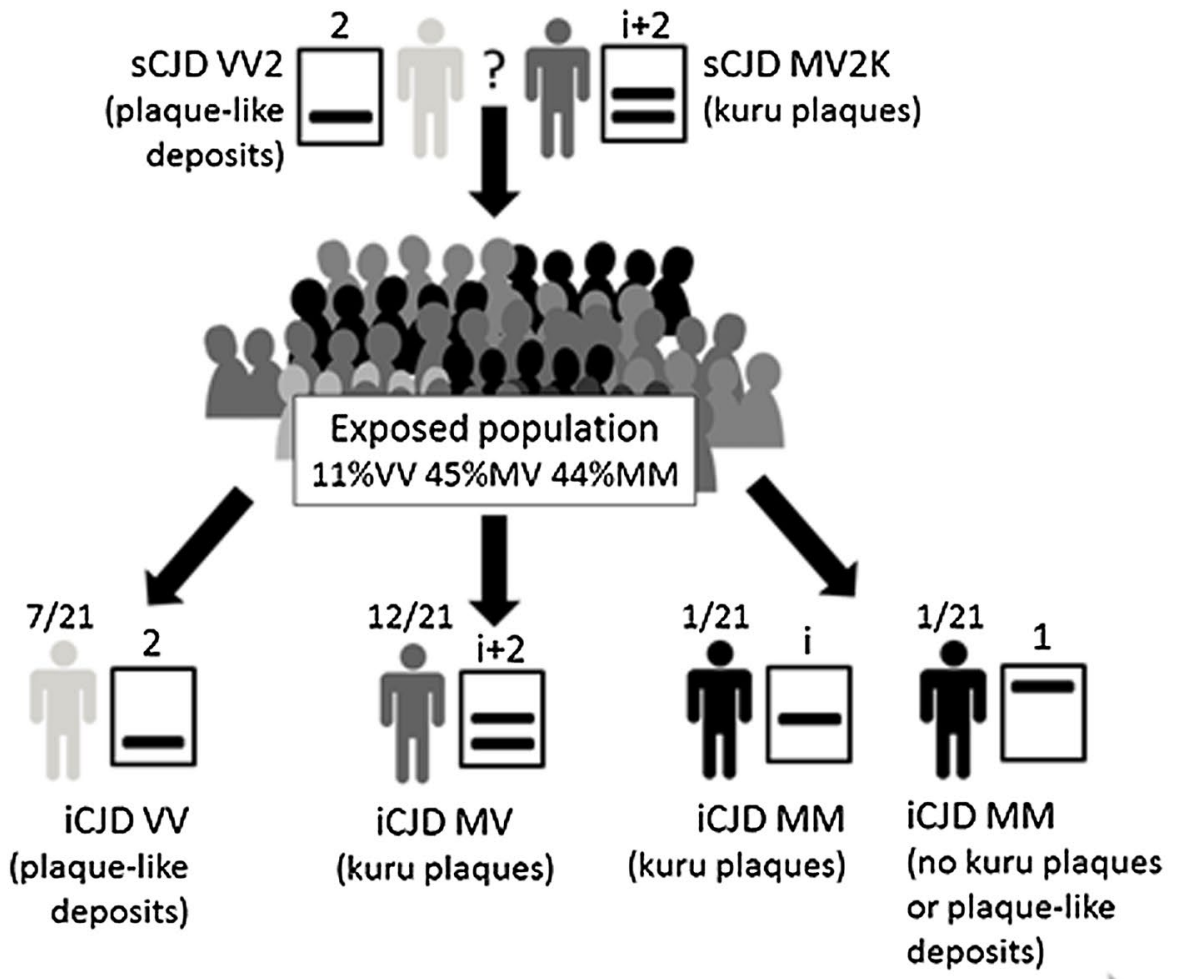

\section{time}

b

3

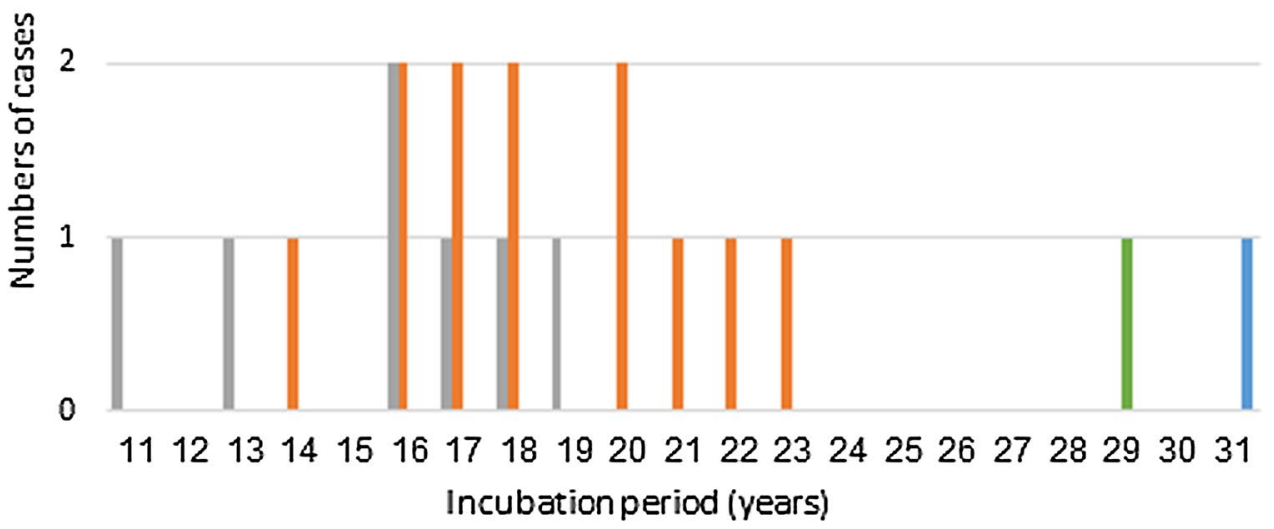

$\square$ V2 $\square$ MVi+2 $\square \mathrm{MMi} \square \mathrm{MM} 1$

\section{Implications for person-to-person transmission}

The RT-QuIC and PMCA comparisons performed here did not support the hypothesis that human-to-human transmission of CJD results in acquired replicative efficiency [36]. Indeed, the RT-QuIC assay showed reduced rather than enhanced seeding activity in hGH-iCJD compared to the matched subtypes of sCJD suggesting that hGH-iCJD is not markedly more of a public health concern than its presumed source, sCJD. However, CJD surveillance systems in countries where contaminated batches of growth hormone were used should remain vigilant for further cases of hGH-iCJD. Some of these cases may be identifiable by the presence of kuru plaques and the presence of $\mathrm{PrP}^{\mathrm{res}}$ type i. However, others cases may appear closely similar to the most common form of sCJD (MM1), and the only 
indication of an iatrogenic aetiology may be in their medical history. The overriding message from the UK experience with hGH-iCJD and perhaps human prion diseases, more generally, is that poorly assessed risks can continue to have ramifications for patients years to decades after the risk source is itself recognised and removed. This point is underscored by a further case of hGH-iCJD being identified during the writing of this report in 2016, some 30 years after treatment with pituitary-derived hGH was abandoned in the UK.

Acknowledgements This report is independent research commissioned and funded by the Department of Health Policy Research Programme and the Scottish Government (PR-ST-0614-00008). Dr Peter Adlard is also funded by the Department of Health. The views expressed in this publication are those of the authors and not necessarily those of the Department of Health or the Scottish Government. The Edinburgh Brain Bank is supported by the Medical Research Council (MRC G0900580). Suzanne Lowrie, Margaret LeGrice and Kimberley Burns are thanked for pathology technical expertise and data retrieval. Jan Mackenzie is thanked for the provision of sporadic CJD PRNP codon 129 genotype data. Biochemical analysis of the dura mater-associated iatrogenic Creutzfeldt-Jakob disease patient specimens was carried out by Ms Emily Wilson. Recombinant human prion protein was generously provided by Dr Gary Mallinson and Dr Dave Jackson (Bristol Institute for Transfusion Sciences, UK). Transgenic mouse brain specimens were a generous gift from Dr Abigail Diack and Professor Jean Manson (Division of Neurobiology, The Roslin Institute, The University of Edinburgh, UK). We would also like to express our gratitude to UK Neuropathologists and their laboratory staff and the relatives of patients for their consent to conduct research on autopsy specimens.

\section{Compliance with ethical standards}

The human tissue examined in this study was provided by the MRC Edinburgh Brain Bank and its use was covered by ethical approval from the East of Scotland Research Ethics Service REC 1 (reference number 16/ES/0084). Informed consent for the research use of autopsy tissue was obtained from the relatives of the deceased whenever necessary. This article does not contain any studies with animals performed by any of the authors.

Conflict of interest The authors declare that they have no conflicts of interest.

Open Access This article is distributed under the terms of the Creative Commons Attribution 4.0 International License (http://creativecommons.org/licenses/by/4.0/), which permits unrestricted use, distribution, and reproduction in any medium, provided you give appropriate credit to the original author(s) and the source, provide a link to the Creative Commons license, and indicate if changes were made.

\section{References}

1. Barria MA, Balachandran A, Morita M, Kitamoto T, Barron R, Manson J et al (2014) Molecular barriers to zoonotic prion transmission. Emerg Infect Dis 20:88-97

2. Bishop MT, Pennington $\mathrm{C}$, Heath $\mathrm{CA}$, Will RG, Knight RSG (2009) PRNP variation in UK sporadic and variant
Creutzfeldt-Jakob disease highlights genetic risk factors and a novel non-synonymous polymorphism. BMC Med Genet 10:146

3. Bishop M, Will RG, Manson JM (2010) Defining sporadic Creutzfeldt-Jakob disease strains and their transmission properties. Proc Natl Acad Sci USA 107:12005-12010

4. Brandel JP, Preece M, Brown P, Croes E, Laplanche JL, Aquid $Y$ et al (2003) Distribution of codon 129 genotype in human growth hormone-treated CJD patients in France and the UK. Lancet 362:128-130

5. Brown P, Brandel JP, Sato T, Nakamura Y, MacKenzie J, Will RG et al (2012) Iatrogenic Creutzfeldt-Jakob disease, final assessment. Emerg Infect Dis 18:901-907

6. Cali I, Miller CJ, Parisi JE, Geschwind MD, Gambetti P, Schonberger LB (2015) Distinct pathological phenotypes of Creutzfeldt-Jakob disease in recipients of prion-contaminated growth hormone. Acta Neuropathol Commun 3:37

7. Collinge J, Clarke AR (2007) A general model for prion strains and their pathogenicity. Science 318:930-936

8. Collinge J, Whitfield J, McKintosh E, Frosh A, Mead S, Hill AF et al (2008) A clinical study of kuru patients with long incubation periods at the end of the epidemic in Papua New Guinea. Philos Trans R Soc Lond B Biol Sci 363:3725-3739

9. Eiden M, Buschmann A, Kupfer L, Groschup MH (2006) Synthetic prions. J Vet Med B Infect Dis Vet Public Health 53:251-256

10. Gibbs CJ Jr, Joy A, Heffner R, Franko M, Miyazaki M, Asher $\mathrm{DM}$ et al (1985) Clinical and pathological features and laboratory confirmation of Creutzfeldt-Jakob disease in a recipient of pituitary-derived human growth hormone. $\mathrm{N}$ Engl $\mathrm{J}$ Med 313:734-738

11. Gibbs CJ Jr, Asher DM, Brown PW, Fradkin JE, Gajdusek DC (1993) Creutzfeldt-Jakob disease infectivity of growth hormone derived from human pituitary glands. N Engl J Med 328:358-359

12. Head MW, Ironside JW, Ghetti B, Jeffrey M, Piccardo P, Will RW (2015) Prion diseases. In: Love S, Budka H, Ironside JW, Perry A (eds) Greenfield's Neuropathology, 9th edn. CRC Press, Boca Raton, pp 1016-1086

13. Heath CA, Barker RA, Esmonde TF, Harvey P, Roberts R, Trend $P$ et al (2006) Dura mater-associated Creutzfeldt-Jakob disease: experience from surveillance in the UK. J Neurol Neurosurg Psychiatry 77:880-882

14. Ironside JW, Knight RSG, Head MW (2011) Iatrogenic Creutzfeldt-Jakob disease. In: Dickson DW, Weller RO (eds) Neurodegeneration: the molecular pathology of dementia and movement disorders, 2nd edn. Wiley-Blackwell, Oxford, pp 381-386

15. Kobayashi A, Asano M, Mohri S, Kitamoto T (2007) Crosssequence transmission of sporadic Creutzfeldt-Jakob disease creates a new prion strain. J Biol Chem 282:30022-30028

16. Kobayashi A, Sakuma N, Matsuura Y, Mohri S, Aguzzi A, Kitamoto $T$ (2010) Experimental verification of the traceback phenomenon in prion infection. J Virol 84:3230-3238

17. Kobayashi A, Iwasaki Y, Otsuka H, Yamada M, Yoshida M, Matsuura Y et al (2013) Deciphering the pathogenesis of sporadic Creutzfeldt-Jakob disease with codon $129 \mathrm{M} / \mathrm{V}$ and type 2 abnormal prion protein. Acta Neuropathol Commun 1:74

18. Kobayashi A, Matsuura Y, Mohri S, Kitamoto T (2014) Distinct origins of dura mater graft-associated Creutzfeldt-Jakob disease: past and future problems. Acta Neuropathol Commun 2:32

19. Kobayashi A, Parchi P, Yamada M, Brown P, Saverioni D, Matsuura Y et al (2015) Transmission properties of atypical Creutzfeldt-Jakob disease: a clue to disease etiology. J Virol 98:3939-3946

20. Kobayashi A, Parchi P, Yamada M, Mohri S, Kitamoto T (2015) Neuropathological and biochemical criteria to identify acquired 
Creutzfeldt-Jakob disease among presumed sporadic cases. Neuropathol. doi:10.1111/neup.12270 (Epub ahead of print)

21. Moda F, Suardis S, Di Fede G, Indaco A, Limido L, Vimercati C et al (2012) MM2-thalamic Creutzfeldt-Jakob disease: neuropathological, biochemical and transmission studies identify a distinctive prion strain. Brain Pathol 22:662-669

22. Moore RA, Head MW, Ironside JW, Ritchie DL, Zanusso G, Choi YP et al (2016) The distribution of prion protein allotypes differs between sporadic and iatrogenic Creutzfeldt-Jakob disease patients. PLoS Pathog 12(2):e1005416. doi:10.1371/journal.ppat.1005416 (eCollection 2016 Feb)

23. Parchi P, Strammiello R, Notari S, Giese A, Langeveld JP, Ladogana A et al (2009) Incidence and spectrum of sporadic Creutzfeldt-Jakob disease variants with mixed phenotype and cooccurrence of $\mathrm{PrP}^{\mathrm{Sc}}$ types: an updated classification. Acta Neuropathol 118:659-671

24. Parchi P, Cescatti M, Notari S, Schulz-Schaeffer WJ, Capellari S, Giese A et al (2010) Agent strain variation in human prion disease: insights from a molecular and pathological review of the National Institutes of Health series of experimentally transmitted disease. Brain 133:3030-3042

25. Parchi P, de Boni L, Saverioni D, Cohen ML, Ferrer I, Gambetti $P$ et al (2012) Consensus classification of human prion disease histotypes allow reliable identification of molecular subtypes: an inter-rater study among surveillance centres in Europe and USA. Acta Neuropathol 124:517-529

26. Peden A, McGuire LI, Appleford NE, Mallinson NE, Wilham JM, Orru CD et al (2012) Sensitive and specific detection of sporadic Creutzfeldt-Jakob disease brain prion protein using realtime quaking-induced conversion. J Gen Virol 93:438-449

27. Peden AH, Sarode DP, Mulholland CR, Barria MA, Ritchie DL, Ironside JW et al (2014) The prion protein protease sensitivity, stability and seeding activity in variably protease sensitive prionopathy brain tissue suggests molecular overlaps with sporadic Creutzfeldt-Jakob disease. Acta Neuropathol Commun 2:152
28. Powell-Jackson J, Weller RO, Kennedy P, Preece MA, Whitcombe EM, Newsom-Davis J (1985) Creutzfeldt-Jakob disease after administration of human growth hormone. Lancet 2:244-246

29. Prusiner SB, Gabizon R, McKinley MP (1987) On the biology of prions. Acta Neuropathol 72:299-314

30. Prusiner SB (1982) Novel proteinaceous particles cause scrapie. Science 216:136-144

31. Rudge P, Jaunmuktane Z, Adlard P, Bjurstrom N, Caine D, Lowe $J$ et al (2015) Iatrogenic CJD due to pituitary-derived hormone with genetically determined incubation times of up to 40 years. Brain 138:3386-3399

32. Shi S, Mitteregger-Kretzschmar G, Giese A, Kretzschmar HA (2013) Establishing quantitative real-time quaking-induced conversion (qRT-QuIC) for highly sensitive detection and quantification of $\mathrm{PrP}^{\mathrm{Sc}}$ in prion-infected tissues. Acta Neuropathol Commun 1:44

33. Swerdlow AJ, Higgins CD, Adlard P, Jones ME, Preece MA (2003) Creutzfeldt-Jakob disease in United Kingdom patients treated with human pituitary growth hormone. Neurology 61:783-791

34. Takeuchi A, Kobayashi A, Parchi P, Yamada M, Morita M, Uno $S$ et al (2016) Distinctive properties of plaque-type dura mater graft-associated Creutzfeldt-Jakob disease in cell-protein misfolding cyclic amplification. Lab Invest 96:581-587

35. The European Creutzfeldt-Jakob Disease Surveillance Network. Diagnostic criteria. http://www.cjd.ed.ac.uk/documents/criteria. pdf. Accessed 06 Sept 2016

36. Xiao X, Yuan J, Qing L, Cali I, Mikol J, Delisle MB, et al (2014) Comparative study of prions in iatrogenic and sporadic Creutzfeldt-Jakob disease. J Clin Cell Immunol 5:pii240 